\title{
The shop around the corner in the Internet age ${ }^{1}$
}

\author{
Jérôme Foncel ${ }^{2}$, Marianne Guyot ${ }^{2}$ et Frédéric Jouneau-Sion ${ }^{3}$
}

\section{Introduction}

Back in the 1990's many analysts predicted that the so-called "new" economy would soon supersede the "old" one. Around-the-corner shops would not survive the Internet age. Yet, ten years later, few remote access firms are profitable. Capacity reductions, LBO and bankruptcies are numerous. In the short run at least, local firms tend to make larger profits and to have larger market shares despite the often aggressive pricing strategies of their "dot com" competitors. ${ }^{4}$ Several explanations have been put forward. In particular, it seems that the public's wariness of on-line payment systems was underestimated. Specific current research programs subsidized by the French government and the European Community are aimed at enhancing secure on-line technologies. In any case, the initial scenario of the analysts, focusing on supply differences in favor of on-line firms, largely underestimated demand effects.

In the long run, remote access technologies are also believed to have geographical impact. There are however very few formalized arguments related to this issue. Some believe that the old shops will tend to serve neighboring customers in downtown areas, whereas on-line firms will serve the outskirts. Others profess massive delocalizations from city centers to suburban areas (see Kotkin, 2001, for a rough survey of the many different arguments). The important growing literature dealing with on-line economy (see e.g Economides, 1996, Matutes and Padilla, 1994, Curien and Oubejja,

1 We are grateful to François Nibart, Régis Renault, Isabel Grilo and Gérard Hamiache for very helpful comments on this work. Comments by the referees and the editor were especially helpful in improving the paper. All remaining errors are ours alone.

2 GREMARS, Université Lille 3.

3 GREMARS, Université Lille 3 and CORE, Université Catholique de Louvain.

4 The inexperience of the managers cannot be the sole explanation. For instance, Hachette Multimedia which does not qualify as a newcomer, has sold its portal to pèrenoël.fr. Another example is the fact that the two French leaders of the on-line market, namely Ooshop and Télémarket - members of the Carrefour and Galeries Lafayette groups respectively - record approximately 6,000 orders a week. Ooshop and Télémarket turnover are approximately 30.49 million Euros and losses are 9.15 and 11.9 million respectively. In comparison, the Carrefour group announces a 64.03 billion turnover (tax excluded) for the year 2000. All these figures stem from the E-marketing letter. 
1999) does not shed much light on possible geographical impacts of the Internet revolution. Clearly a formal treatment of this question calls for a genuine spatial competition model.

We propose to study the competitive behavior of an ordinary shop facing a remote access competitor. We propose a variant of Hotelling's framework, the famous "linear city" model. In choosing the local firm, the customer incurs a linear transportation cost proportional to the distance between her location and the local firm. Choosing the on-line firm induces a fixed cost whatever the location of the customer. This fixed cost may represent "objective" monetary costs (for instance purchase of hard- and software or fixed shipping fees). It may also include a monetary equivalent for wariness of on-line transactions.

Although the main mechanisms of our model stem from the demand differences described above, it also copes with supply comparative advantages. Indeed, many analysts emphasize the cost reductions implied by online technology and this seems particularly true in the service sector. When production costs are linear, the difference between the fixed cost incurred by the use of the on-line technology and the possible supply advantage of the on-line firm is a key parameter of the model and is at the core of the analysis.

In the short run, the location of the ordinary firm is fixed and the two firms compete on prices. The market allows for three types of equilibria. First, when the key parameter is sufficiently large and/or the location is close to the center, the local firm enjoys a monopoly position. Second, we identify two types of duopoly. In the first case (hereafter type-I duopoly), the on-line firm's customers are located either on the left or on the right of the town. In the second case (type-II duopoly), the on-line firm serves extreme right and left customers while the local firm's customers are all located in the center. The type-I duopoly is by far the most probable one. Type II duopoly occurs only if the fixed cost is sufficiently small and the location is close to the center. We also show that the local firm can never be driven out of the market by its competitor unless the key parameter is negative. Thus for the new economy to supersede the old economy, we need the cost advantages of the on-line firm to compensate for its demand disadvantage. We also show that in general the most aggressive pricing rule is always chosen by the firm which enjoys the smallest market share and the lowest profit. Our model thus accounts for some of the stylized facts mentioned above.

In the long run, location becomes strategic for the local firm. Long run equilibria are difficult to study for, as usual in Hotelling's setting, short run equilibria may fail to exist for some values of the fixed cost (this feature has been emphasized in the Hotelling model by D'aspremont, Gabsciewicz and Thisse, 1979). As Obsbourne and Pitchick (1987) we then complete our 
analysis by looking for "simple" mixed equilibria. We show that when the key parameter is larger than a threshold, mixed equilibria in which only the on-line firm randomizes exists. For sufficiently large values of the key parameter, the local firm is better off in the center of the town, enjoying a monopoly position. When the key parameter decreases the local firms moves toward the edge of the town (either left or right due to symmetry). Our model then gives some credit to the "delocalization scenario" in which the local firm ultimately leaves the center to outlying areas as the competitiveness of the on-line firm increases (see Kotkin, 2001).

Our paper can be compared to the approach followed by Degryse (1996) and Bouckaert and Degryse (1995). They are, however, several important differences. First, in our paper the remote access service is offered by a single firm. The idea is to consider specialized firms (such as for instance Amazon.com versus an "around-the-corner" book shop). This allows us to emphasize the competition between "local" and "on-line" firms. Second, the proportion of remote access contracts is endogenously derived. Basically, we attempt to explain the relatively small market shares of the new economy firms. Balsubramanian (1998) and Bouckaert (2000) also investigate the issue of competition between an on-line firm and possibly several local firms. Some of their results are related to ours. For instance, they also find that the online firm enjoys a smaller market share despite aggressive pricing. This occurs only when the fixed cost is large enough. However, they adopt a circular city framework. In these models, the local firm's locations are symmetrical and genuine spatial aspects cannot be handled. As a consequence, their models are unable to provide information on the issue of the geographical impact of new technologies.

The rest of the paper goes as follows. Section 2 sets the model and presents short run equilibria. Long run equilibria are studied in section 3 . Section 4 concludes. Computations may be found in the Appendix Section.

\section{The model}

We consider two firms selling a single indivisible good. The first one is a corner shop, hereafter referred to as the "local firm". This firm chooses a price $p \geq 0$. If a consumer chooses this firm, she incurs a transportation cost. Transportation costs are assumed to increase linearly with the distance between the local firm and the customer. ${ }^{5}$ Consumers are assumed to be uniformly distributed on the interval [0, 1] as in Hotelling (1929). As usual in this kind of setting, note that "space" may not have the usual geographical

5 The difficulties involved in the computation of the equilibrium for more general transportation cost functions are the main argument for the linearity assumption. 
interpretation. Hotelling's device is mainly a way of incorporating horizontal differentiation among customers. However, as our paper also focuses on geographical issues, there is no harm in adopting an "intuitive view" of the "space".

Formally, if we consider a consumer whose address is $x \in[0,1]$, her indirect utility level when she chooses the local firm is:

$$
U=-p-t\left|x-x_{a}\right|,
$$

where $x_{a} \in[0,1]$ stands for the address of the local firm and $t>0$ the transportation cost per unit of distance. Without loss of generality, the surplus (gross of price and transportation costs) derived from the consumption of the good is normalized to zero. Each consumer is assumed to be interested in a single unit of good. When this consumer purchases the same good online, her indirect utility is defined by

$$
U^{*}=-p^{*}-F^{*}
$$

with $p^{*} \geq 0$ the on-line firm price. The fixed "cost" $F^{*}>0$ includes connection costs, the technological burden, etc. More generally, if we consider the customer located at the same address as the local firm (that is, the one with no transportation costs), $F^{*}>0$ represents a disadvantage related to the remote access service. This cost may include some wariness of these services.

We denote $\underline{U}$ the level of indirect utility when the consumer does not consume the good. As we normalized the surplus of the good to zero, it is required that $\underline{U}<0$ otherwise the demand for the good is zero. In IO models, this kind of reservation utility is often provided by the presence of an outside good providing a fixed level of indirect utility. In cases of spatial competition, the question is linked to the so-called "covered market" issue. We assume that $\underline{U}$ is low enough (say $\underline{U}=-\infty$ ) so that each consumer is served in any equilibrium.

\subsection{Profit functions}

A necessary and sufficient condition for a customer whose address is $x$ to choose the on-line firm is:

$$
-p-t\left|x-x_{a}\right| \leq-p^{*}-F^{*}
$$

Clearly, the problem allows for a symmetry. We shall deal with the case $0 \leq x_{a} \leq \frac{1}{2}$ only. The case $1 \geq x_{a}>\frac{1}{2}$ can be deduced by a change of variables $y_{a}=1-x_{a}$.

We assume that the consumers are all endowed with the same amount of money to purchase the good. Considering that consumers are uniformly 
distributed on $[0,1]$ we normalize total demand to 1 . Using condition (1) the market share of the local firm is then given by:

$\Delta\left(x_{a}, p, p^{*}, F^{*}, t\right)=\left\{\begin{array}{lll}0 & \text { if } & p \geq p^{*}+F^{*} \\ 2 \frac{p^{*}+F^{*}-p}{t} & \text { if } & p^{*}+F^{*} \geq p \geq p^{*}+F^{*}-t x_{a} \\ x_{a}+\frac{p^{*}+F^{*}-p}{t} & \text { if } & p^{*}+F^{*}-t x_{a} \geq p \geq p^{*}+F^{*}-t\left(1-x_{a}\right) \\ 1 & \text { if } & p \leq p^{*}+F^{*}-t\left(1-x_{a}\right)\end{array}\right.$

The market share of the on-line firm is $\Delta^{*}\left(x_{a}, p, p^{*}, F^{*}, t\right)=$ $1-\Delta\left(x_{a}, p, p^{*}, F^{*}, t\right)$. Note that the market share of the local firm may be rewritten as

$\Delta\left(x_{a}, p, p^{*}, F^{*}, t\right)=\left\{\begin{array}{lll}0 & \text { if } & p-c \geq p^{*}-c^{*}+F^{*}-\epsilon \\ 2 \frac{p^{*}-c^{*}-(p-c)+\left(F^{*}-\epsilon\right)}{t} & \text { if } & p^{*}-c^{*}+F^{*}-\epsilon \geq p-c \\ & \text { and } p-c \geq p^{*}-c^{*}+F^{*}-\epsilon-t x_{a} \\ x_{a}+\frac{p^{*}-c^{*}-(p-c)+\left(F^{*}-\epsilon\right)}{t} & \text { if } \quad p^{*}-c^{*}+F^{*}-\epsilon-t x_{a} \geq p-c \\ & \text { and } p-c \geq p^{*}-c^{*}+F^{*}-\epsilon+t\left(1-x_{a}\right) \\ 1 & \text { if } p-c \leq p^{*}-c^{*}+F^{*}-\epsilon+t\left(1-x_{a}\right)\end{array}\right.$

where $c$ (respectively $c^{*}>0$ ) represents the marginal cost of the local firm (resp. the on-line firm). The parameter $\varepsilon=c-c^{*}$ stands for the cost advantage of the on-line firm (if $\varepsilon>0$ ). Thus $\Delta\left(x_{a}, p, p^{*}, F^{*}, t\right)=\Delta\left(x_{a}, p-c\right.$, $\left.p^{*}, p^{*}-c^{*}, F^{*}-\varepsilon, t\right)$. It is easily seen that the same result applies to the market share of the on-line firm.

Now the profit of the local firm is $\pi\left(x_{a}, p, p^{*}\right)=(p-c) \Delta\left(x_{a}, p, p^{*}, F^{*}, t\right)=(p-c) \Delta\left(x_{a}, p-c, p^{*}-c^{*}, F^{*}-\varepsilon, t\right)$

Similarly, we have for the on-line firm

$$
\begin{gathered}
\pi^{*}\left(x_{a}, p, p^{*}\right)=\left(p^{*}-c^{*}\right) \Delta^{*}\left(x_{a}, p, p^{*}, F^{*}, t\right)= \\
\left(p^{*}-c^{*}\right) \Delta^{*}\left(x_{a}, p-c, p^{*}-c^{*}, F^{*}-\varepsilon, t\right)
\end{gathered}
$$

This simple computation suggests that the strategic variables of the model should be the markups (that is $p-c$ and $p^{*}-c^{*}$ ) instead of the prices and $F^{*}-\varepsilon$ instead of $F^{*}$. Now if the cost differences do more than offset the fixed cost we may have $F^{*}-\varepsilon<0$. Another consequence of this change in the variables is that we can consider the case where $c=c^{*}=0$, as long as we agree that $p$ and $p^{*}$ must be understood as markups and $F^{*}$ as a "supply adjusted" demand effect that can be negative.

Another interpretation of negative values for $F^{*}$ is the following. ${ }^{6}$ Assume that there is also a fixed disutility to shopping outside (it is inconvenient to leave the house, regardless of the distance to the store). The

6 We are indebted to an anonymous referee for this suggestion. 
parameter $F^{*}$ would then be interpreted as the difference between both fixed costs. To put it differently, $F^{*}$ is then a net demand effect. If the disutility of the local firm is strong enough then $F^{*}$ may be negative.

Finally, it is worth noting that shipping costs are involved in on-line purchases. Usually, shipping cost as a percentage of total order amount decreases significantly with increasing total order amount. In our model, since customers have unit demand it is not clear whether we should assume shipping costs to be part of the marginal cost of the on-line firm or of the fixed cost borne by customers. Indeed, as proposition 3 makes clear, the effect on equilibria is not the same. Inclusion of shipping costs has no effect on the local firm's price, whereas it does on the on-line firm's price. When shipping costs are included in the marginal cost rather than in the fixed cost, the on-line firm's price is higher. In the real world, the way on-line firms pass these costs on to customers is part of their strategy. This issue remains open and is beyond the scope of this paper.

\subsection{Short run best responses}

In the short run, both firms simultaneously choose their own price in a noncooperative way. The local firm may change its address in the long run only (see Section 3). Moreover, we restrict pricing to pure strategies.

The best response of the local firm is given by the following proposition:

Proposition 1. The local firm best response is

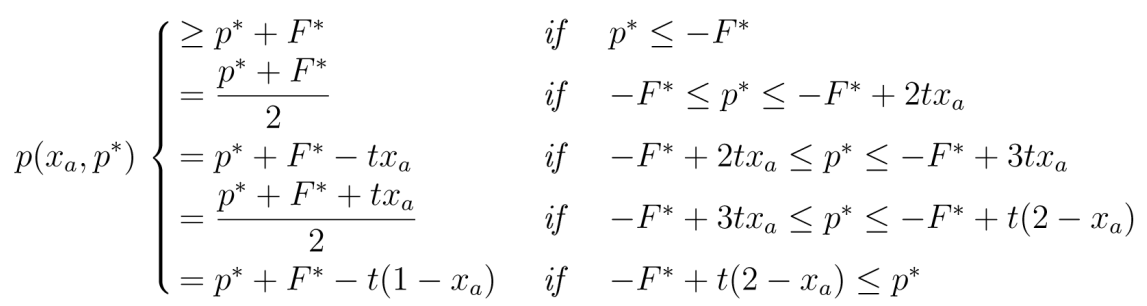

Proof : See Appendix 1.

Notice that the first case corresponds to a potential online monopoly situation. This case can only occur when $F^{*}<0$. When the local firm chooses to produce, comparative static effects are consistent with the intuition. The markup $p\left(x_{a}, p^{*}\right)$ increases with that of its rival and with $F^{*}$. The effect of the transportation cost is ambiguous. This is not surprising, since a transportation cost increase relatively improves the utility of on-line services. This should force the local firm to propose a more attractive price. However, the remaining customers of the local firm are more "captive" than before. This last effect may cause the price to increase. 
As the computation in Appendix 1 makes clear, the price response of the local firm is not always derived from the first order condition (its profit function is not everywhere differentiable). Indeed, when $-F^{*}+2 t x_{a} \leq p^{*} \leq$ $F^{*}+3 t x_{a}$ the profit function is strictly increasing (resp. decreasing) when $p$ is smaller (resp. larger) than $p^{*}+F^{*}-t x_{a}$ Note that in this case, $\partial p\left(x_{a}, p^{*}\right) / \partial p^{*}=1$. This means that the competition is tighter (this fact will have important consequences, see section 2.3 for details). sition:

We now give the best response of the on-line in the following propo-

Proposition 2. The best response of the on-line firm is given by

i) if $0 \leq x_{a} \leq \frac{3}{2}-\sqrt{2}$

$$
p^{*}\left(x_{a}, p\right)\left\{\begin{array}{lll}
=p-F^{*} & \text { if } & p \geq F^{*}+t\left(1-\sqrt{x_{a}}\right)^{2} \\
=\frac{p-F^{*}+t\left(1-x_{a}\right)}{2} & \text { if } & F^{*}+t\left(1-\sqrt{x_{a}}\right)^{2} \geq p \geq F^{*}-t\left(1-x_{a}\right) \\
\geq p-F^{*}+t\left(1-x_{a}\right) & \text { if } & p<F^{*}-t\left(1-x_{a}\right)
\end{array}\right.
$$

ii) if $\frac{3}{2}-\sqrt{2} \leq x_{a} \leq \frac{1}{2}$

$$
p^{*}\left(x_{a}, p\right)\left\{\begin{array}{lll}
=p-F^{*} & \text { if } & p \geq F^{*}+\frac{t}{2} \\
=\frac{p-F^{*}+\frac{t}{2}}{2} & \text { if } & F^{*}+\frac{t}{2} \geq p \geq F^{*}-t\left((\sqrt{2}+1) x_{a}-\frac{1}{\sqrt{2}}\right) \\
=\frac{p-F^{*}+t\left(1-x_{a}\right)}{2} & \text { if } & F^{*}-t\left((\sqrt{2}+1) x_{a}-\frac{1}{\sqrt{2}}\right) \geq p \geq F^{*}-t\left(1-x_{a}\right) \\
\geq p-F^{*}+t\left(1-x_{a}\right) & \text { if } & p<F^{*}-t\left(1-x_{a}\right)
\end{array}\right.
$$

Proof : See Appendix 2.

Note that when the on-line firm produces, its best response is a discontinuous function of $p$. Indeed, in case $i$ ), it is easily checked that if $x_{a}>0$ we have $p-F^{*} \neq \frac{p-F^{*}+t\left(1-x_{a}\right)}{2}$ when $p=F^{*}+t\left(1-\sqrt{x_{a}}\right)^{2}$. In a similar fashion, in case $i i) \frac{p-F^{*}+\frac{t}{2}}{2} \neq \frac{p-F^{*}+t\left(1-x_{a}\right)}{2}$ when $p=F^{*}-t\left((\sqrt{2}+1) x_{a}\right.$ $\left.-\frac{1}{\sqrt{2}}\right)$ and $x_{a}<1 / 2$. This discontinuity is a consequence of the non-concavity of the on-line firm's profit function (with respect to $p^{*}$ ). This feature is worth noting since it has dramatic consequences for the equilibria. Indeed, assume we start from a relatively high value of $p^{*}$ so that the online firm's customers are all located at the right side of the city (as described in Figure 2.1) 
Figure 2.1. Type-I duopoly

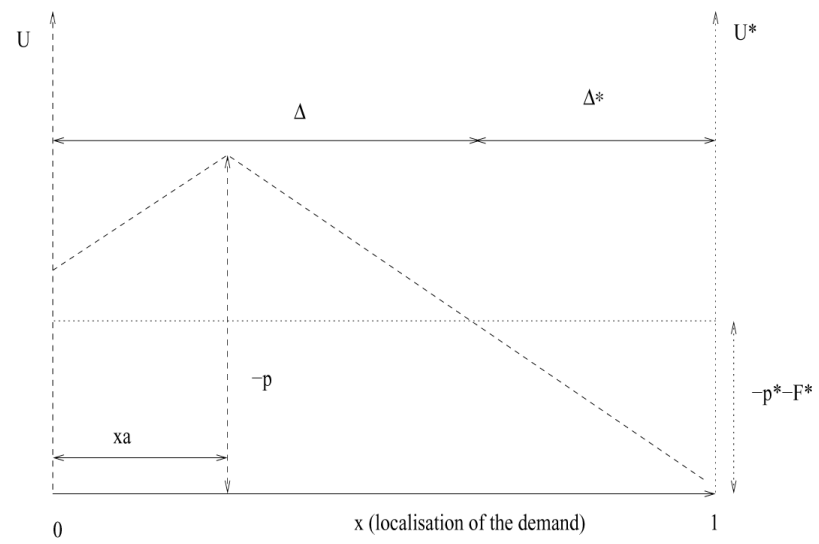

In this case, we have $\partial \Delta^{*}\left(x_{a}, p, p^{*}, F^{*}, t\right) / \partial p^{*}=-1 / t$ and the profit function is (locally) a quadratic concave function of $p^{*}$. Hereafter, we refer to this case as type-I duopoly.

If $p^{*}$ decrease, we ultimately reach another case (as described in Figure 2.2) in which the on-line firm captures far left and right customers. In this case, $\partial \Delta^{*}\left(x_{a}, p, p^{*}, F^{*}, t\right) / \partial p^{*}=-2 / t$ and the profit function is (locally) a quadratic concave function of $p^{*}$ (different from the previous one). Hereafter, we refer to this case as type-II duopoly.

Figure 2.2. Type-II duopoly

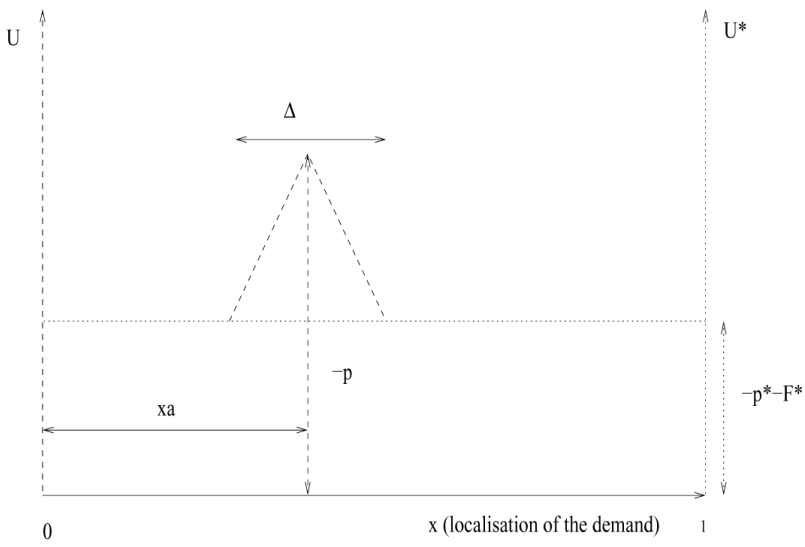

It may be that both regimes display a local maximum. In this case, the online firm has to choose between a "soft" pricing strategy (leading to type-I duopoly) or a more aggressive one (leading to type-II duopoly). Figure 2.3 presents a case in which the "aggressive" pricing strategy corresponding to the type-II duopoly is best. 
Figure 2.3. Example of a non-concave profit function for the on-line firm

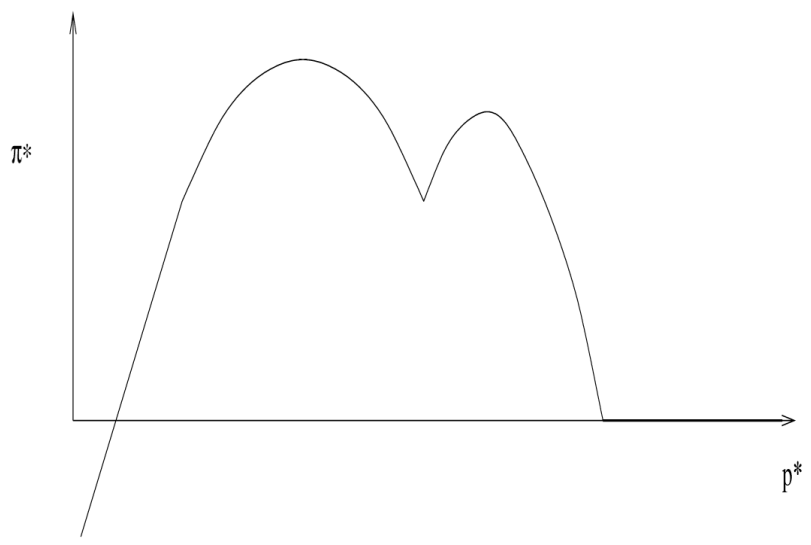

Yet a small change in parameters $F^{*}, t$ or $x_{a}$ can cause a jump in the global maximum from one type of duopoly to the other, hence the discontinuity in the on-line firm's best response. Note that a similar problem arises in the commonly-used Hotelling's model. Indeed, as stressed by d'Aspremont et al. (1979) when the firms are close enough, two pricing strategies are possible (an "aggressive" strategy and a "soft" strategy). In Hotelling's model, this leads to discontinuous response functions. Yet, in our framework the best response function of the on-line firm is discontinuous whereas the best response function of the local firm is continuous but does not always derive from a first order condition.

There are cases (more precisely $p=F^{*}+t\left(1-\sqrt{x_{a}}\right)^{2}$ when $0 \leq x_{a}$ $\leq \frac{3}{2}-\sqrt{2}$ and $p=F^{*}-t\left((\sqrt{2}+1) x_{a}-\frac{1}{\sqrt{2}}\right)$ when $\frac{3}{2}-\sqrt{2} \leq x_{a} \leq$ $1 / 2$ ) where the best possible choice of $p^{*}$ for both strategies leads to the same level of profit. In these cases, the on-line firm can choose either to serve only the right edge of the city with a -relatively- high $p^{*}$ or to cut its price and serve two separately located groups of customers. This will be of particular importance in section 3. Note that this arises very naturally as soon as geographical aspects are seriously considered. Indeed, as figures 2.1 and 2.2 make clear, it depends on the presence of "borders" for the linear city (this feature cannot be captured by a circular city model in which type-I duopoly never exists).

\subsection{Pure strategy short run equilibria}

Nash equilibria directly follow from both firms' best responses. In the remaining of the paper we restrict our analysis to the case $F^{*}>0$. The other case $F^{*}<0$ requires extremely cumbersome computations and leads to the 
impossibility of characterizing all equilibria. We thus restrict our attention to situations in which the online firm is not capable to drive the traditional firm out of the market. This is quite realistic (even in the context of Section 3 where the location is endogenously derived) if we refer to the period described in the introduction.

Proposition 3. We have three pure strategy short run equilibria:

only if

1. type-I duopoly: $p=\frac{F^{*}-t\left(1+x_{a}\right)}{3}$ and $p^{*}=\frac{t\left(2-x_{a}\right)-F^{*}}{3}$ if and

$$
\max \left(0 ; \frac{1}{2}-\frac{3}{2 \sqrt{2}}+x_{a}\left(\frac{3}{\sqrt{2}}+2\right)\right) \leq F^{*} / t \leq 2-x_{a}
$$

2. type-II duopoly: $p=\frac{F^{*}+t / 2}{3}$ and $p^{*}=\frac{t-F^{*}}{3}$ if and only if

$$
0 \leq \frac{F^{*}}{t} \leq\left(\frac{3}{\sqrt{2}}+\frac{3}{2}\right) x_{a}-\frac{3}{2 \sqrt{2}}+\frac{1}{4}
$$

3. Local firm monopoly: $p=F^{*}-t\left(1-x_{a}\right)$ if and only if

$$
F^{*} / \geq 2-x_{a} \text { or }\left\{1 \leq F^{*} / t \leq 3 / 2 \text { and } x_{a}=1 / 2\right\}
$$

Proof : See Appendix 3.

A graphic representation of the above proposition is provided by figure 2.4

Figure 2.4. Short run equilibria

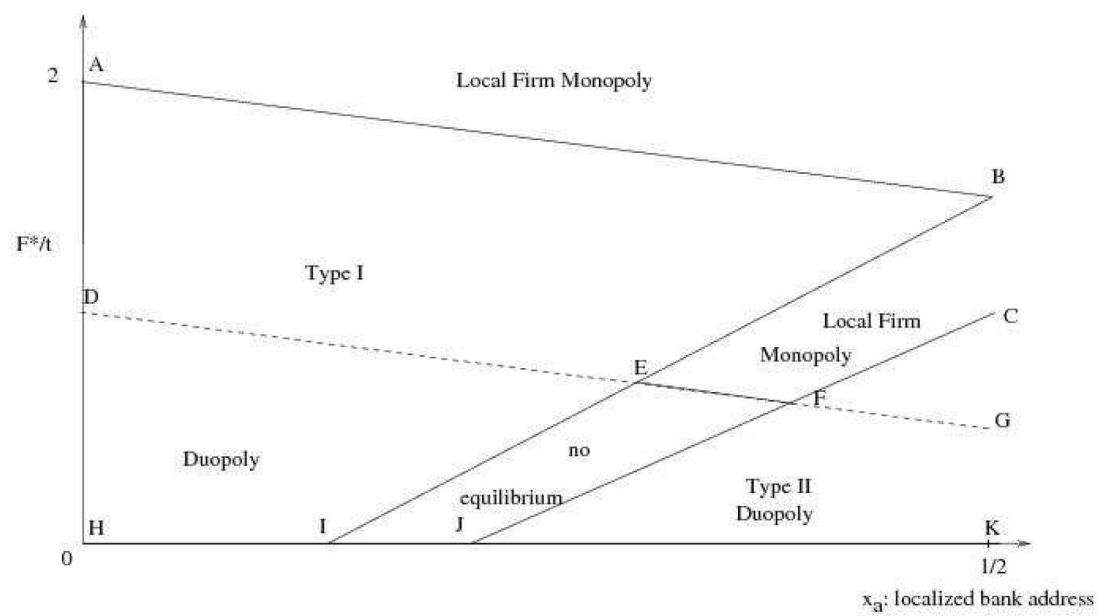


We now provide several intuitive interpretations of these results. Two distinctions will be of particular importance here:

i) the distinction proposed by Bain (1956), namely that of blockaded, deterred and accommodated entry;

ii) the distinction between the two types of duopoly that can be generated by our model.

i) Assume first that $F^{*} / t$ is large. The on-line firm is prevented from entering the market. Indeed $p^{*}$ needs to be very low to capture the worse-off customer of the local firm (note that as $x_{a} \leq 1 / 2$ this customer is located at address $x=1$ ). Ultimately, such a price is negative and the on-line firm is kept out of the market and does not compete directly with the local firm. This corresponds to the "blockaded entry" case of Bain's taxonomy. Graphically, the frontier of the "blockaded entry" case is the line AB of figure 2.4. Note that as the welfare of the customer located at $x=1$ increases with $x_{a}$, blockaded entry is easier when $x_{a}$ is closer to $1 / 2$. That is, lower levels of $F^{*}$ can sustain blockaded entry as $x_{a}$ increases. Thus, line AB has a negative slope.

Assume now that $F^{*} / t$ is smaller, so that by a similar argument the local firm cannot sustain a monopoly: the compensation of its worse-off customer for very high transportation cost will ultimately lead to a negative $p$. Thus it is impossible to deter entry. This is exactly what happens below line DEFG (the equation of this line is $\frac{F^{*}}{t}=1-x_{a}$ ) Thus, below this line, the monopoly strategy is not a credible threat for the local firm. Line DEFG has a negative slope with respect to $x_{a}$ for the same reason as above. Intermediate cases exist (between $\mathrm{AB}$ and $\mathrm{DEFG}$ ), where the local firm can deter or accommodate entry. In our set-up, both outcomes emerge as equilibria.

ii) To understand why several equilibria can coexist for similar values of $F^{*} / t$ when $x_{a}$ moves from left to central position, we should recall from section 2.2 that, as a consequence of the "geography" of our model, the profit function of the on-line firm may not be concave. First assume $x_{a}$ is low. It will then be very costly for the on-line firm to capture far-left customers (ultimately, $p^{*}$ would be negative) and type-II duopoly cannot occur. This situation corresponds to the left side of the IEB boundary. In figure 2.3 above, it entails the non-existence of the left local maximum of the profit function of the on-line firm. Similarly, when the location of the local firm is central $\left(x_{a}=1 / 2\right)$, there is no way to capture right-edge customers only. A continuity argument makes it clear that type-I duopoly cannot occur when the location of the local firm is close to $1 / 2$. Thus, when $x_{a}$ is large enough, the right local maximum of the above figure 2.3 disappears. Hence type-I duopoly cannot appear on the right side of the JFC line. 
Recall from proposition 1 that the slope of $p$ as a function of $p^{*}$ is either 1 or $1 / 2$. A sensitivity of 1 corresponds roughly to the "Bertrand" case of "hard" competition, whereas a sensitivity of $1 / 2$ corresponds to a softer form of competition (as in a duopoly Cournot case, for instance). A closer look at the proofs reveals that the "hard" form of competition arises exactly when the on-line firm's profit is not concave, that is, between the JFC and the IEB lines. Hence if we consider the cases where a single type of duopoly can coexist with a monopoly (here we consider regions ABED and CFG) the pricing strategy is always soft and accommodated entry prevails. Conversely, between the JFC and the IEB lines the pricing competition is always tough and deterred entry is the rule. Of course only the strongest firm survives, i.e. the local firm in the BEFC region.

Finally, between the JFC and the IEB lines when deterred entry is impossible (that is in the JIEF region) no pure strategy equilibrium exists. This can be viewed as a consequence of proposition 2 . Recall indeed that the best response $p^{*}\left(x_{a}, p\right)$ is not continuous. This gap may result in no intersection between the best response functions. This final case is very similar to the non-existence in the pricing game of the seminal Hotelling paper.

Note that the values of $F^{*} / t$ and $x_{a}$ play a leading role for both arguments (i and ii). Consequently, moving from left to right position (keeping $F^{*} / t$ fixed) or "top-down" (keeping $F^{*} / t$ fixed) may induce different types of equilibria.

\subsection{Links with stylized facts in the short run}

The results of the pricing game are consistent with what happened during the "new economy" recession. First, on-line firms then typically enjoyed rather small market shares. Second, this was true even though "new economy" firms offered attractive prices (such as, for instance, for banking services) and enjoyed comparative supply advantages. Third, profits of on-line firms turned out to be smaller than those of local firms. A high enough $F^{*} / t$ leads to the apparently paradoxical coexistence of these facts. If we interpret $F^{*}$ as arising from a certain wariness of on-line transactions, actual pricing rules are consistent with a substantial value of $F^{*} / t$. To see this, consider the results below (which are easily derived):

- The on-line firm's profits and market share are higher than those of the local firm if and only if one of the two conditions is fulfilled: the type-I duopoly prevails and $\frac{F^{*}}{t} \leq \frac{1}{2}-x_{a}$ or the type-II duopoly prevails and $\frac{F^{*}}{t} \leq \frac{1}{4}$ 
- The markup of the on-line firm is higher than that of the local firm if and only if one of the two conditions is fulfilled : the type-I duopoly prevails and $\frac{F^{*}}{t} \leq \frac{1}{2}-x_{a}$ or the type-II duopoly prevails and $\frac{F^{*}}{t} \leq \frac{1}{6}$

We note that the on-line firm's profits and market share are lower than those of the local firm, except when there are low levels of $F^{*} / t$. Indeed, a high fixed cost $F^{*}$ relative to $t$ gives local market power to the local firm. As a consequence, if the on-line firm ever produces, it should choose a smaller mark-up (and also a smaller price if this firm enjoys a supply comparative advantage). This effect is reinforced if the location of the local firm is close to the center. When $F^{*} / t$ is large, this effect is strong enough to reduce the market share of the on-line firm even though its pricing is more aggressive (obviously, profits are also lower). As $F^{*} / t$ decreases, the comparative disadvantage is not strong enough and the on-line firm has an easier time. Note that in our setting, the highest profit is always realized by the firm with the highest markup. Now, if the supply advantage of the online firm is strong enough, it may well choose a more attractive price even though it is more profitable (compared to its competitor).

Note that caution is required when correlating concentration on a downtown market with low market share and profits, as usually suggested by the popular press. Indeed in the type-II duopoly case, the local firm's profits and market share may be higher than those of the on-line firm. In any case, a large market share for the on-line firm cannot follow solely from demand conditions, as long as $F^{*}>0$. Conditions of relatively advantageous supply (notably weaker production costs and/or partial vertical competition) have to counterbalance the effects described above.

\subsection{Fixed cost differences}

Up to now we have only considered (linear) variable cost differences. Proposition 3 can also be considered in the case of fixed cost differences. Indeed, it is often claimed that on-line firms enjoy substantial cuts in real-estaterelated, and/or storage costs.

Assume first that fixed costs are low enough that entry decisions are not affected. Fixed cost differences only affect profits. For instance, the local firm enjoys higher profits -fixed costs excluded- and the reverse holds when fixed costs are included. This would probably be the case if $\pi^{*}$ and $\pi$ are close to each other (fixed costs excluded) and if the fixed costs incurred by the on-line firm are lower than those of the local firm.

A more interesting case arises when fixed costs are high. Obviously, this can affect the result, making it impossible for the on-line firm to keep the local firm out of the market when $F^{*}>0$. If $G^{*}$ (resp. $G$ ) stands for the 
on-line (resp. local) firm's fixed costs, the monopoly of the on-line firm is possible if and only if

$$
p \Delta\left(x_{a}, p, p^{*}, F^{*}, t\right)<G \forall p \Rightarrow p^{*}>G^{*}
$$

Easy computations, using proposition 1, show that this condition is equivalent to $-F^{*}+t\left(2-x_{a}\right)>G^{*}$ and $G>t$. Thus, if the fixed costs incurred by the on-line (resp. local) firm are low (resp. high) enough, the on-line firm can maintain a monopoly.

\section{$3 \quad$ Long run equilibria}

According to the usual approach to long run equilibria in Hotelling's models, we should now consider a two-stage game in which the location is chosen first before price competition takes place. Blindly applying this principle in our setting would, however, lead us to maximize profits for the local firm with respect to $x_{a}$ for fixed values of $F^{*} / t$. This approach cannot be directly applied here. Indeed, if we keep $F^{*}$ and $t$ fixed, there are values of $x_{a}$ such that no short run equilibrium exists. Thus the equilibria derived by the previous technique cannot be shown to be sub-game perfect. Indeed, for the solution to be sub-game perfect we must investigate any first step deviation and show that the second step equilibrium would always make the local firm worse-off. But this cannot be shown if some choices of $x_{a}$ result in an absence of (second-step) equilibrium.

\subsection{Short run mixed equilibria}

A possible solution is to extend the set of strategies so that the short run equilibrium always exists. Indeed, since the payoff functions are continuous, we know (see Glicksberg, 1952) that a mixed strategy equilibrium exists for any value of $F^{*}, t$ and $x_{a}$.

The problem is that mixed strategy equilibria are typically difficult to derive in Hotelling-type models. For the initial Hotelling's model the best results (to our knowledge) have been obtained by Osbourne and Pitchik (1987). They provide a partial analysis of the mixed equilibria. More precisely they study a class of mixing strategies and provide a taxonomy of some possible mixed equilibria. As they mention, they cannot come up with a complete study of the mixed equilibria, which means that the genuine long run solutions may not coincide with their proposal.

Compared to Osbourne and Pitchik (1987) our problem is more complex because the market share of the on-line firm may be the union of two disjoints intervals (this typically happens in the type-II duopoly case). As 
a consequence, we may have two indifferent consumers. Now the paper by Osbourne and Pitchik (1987) makes explicit use of the fact there is only one indifferent consumer. We then have to adapt their approach to our model.

More precisely we study some "simple" mixed strategies in which only the on-line firm uses non degenerate pricing rules. The on-line firm then randomizes over a set of two distinct prices, one of which corresponds to a type-I and the other a type-II duopoly. In the following proposition, we characterize the region of the space $\left(F^{*} / t, x_{a}\right)$ where these strategies provide mixed equilibria.

Proposition 4. When the firms can randomize their prices the short run game allows for equilibria 1 to 3 of proposition 3 and a mixed duopoly equilibrium such that

$$
\begin{aligned}
& p=F^{*}-t\left((\sqrt{2}+1) x_{a}-1 / \sqrt{2}\right) \\
& p^{*} \in\left\{t \frac{\sqrt{2}+1}{4}\left(1-2 x_{a}\right), t \frac{\sqrt{2}+2}{4}\left(1-2 x_{a}\right)\right\} \\
& \mathbf{P}\left(\tilde{p}^{*}=t \frac{\sqrt{2}+1}{4}\left(1-2 x_{a}\right)\right)=\frac{t x_{a}(6+4 \sqrt{2})+t(\sqrt{2}-3)-2 \sqrt{2} F^{*}}{2 \sqrt{2} F^{*}-t x_{a}(6+2 \sqrt{2})+3 t}
\end{aligned}
$$

with the necessary and sufficient conditions:

$$
\begin{aligned}
& x_{a}(3 / \sqrt{2}+2)-\frac{3}{2 \sqrt{2}}+\frac{1}{2} \geq \frac{F^{*}}{t} \geq x_{a}(3 / \sqrt{2}+3 / 2)-\frac{3}{2 \sqrt{2}}+\frac{1}{4} \\
& F^{*} / t \geq 0 \\
& \text { Proof : See Appendix } 4 .
\end{aligned}
$$

These results can be directly interpreted on Figure 2.4. It is easily seen that the (random) outcomes of the mixed strategy equilibria are either type-I or type-II duopolies (region IEFJ). Moreover the probability of reaching a type-I (resp. type-II) duopoly goes to 1 when approaching the IEB (resp. JFC) line. We also notice that the deterred entry monopoly coexists with the mixed equilibrium in region EBCF (for the local monopoly). The other areas display the same equilibria as in Section 2.3. Finally, enlarging the set of strategies of the on-line firm gives it a somewhat easier time. However, as simple calculations show, the most aggressive pricing choice always coincides with the lowest profit ${ }^{7}$.

\subsection{Long run solutions}

The computation of the long run equilibrium is straightforward and results from a simple comparison of the (expected) profits of the local firm when $x_{a}$ is optimized. Results are presented below and comments follow.

7 This deserves more precise comment, for the market share, profits and price of the on-line firm are now considered as random. This result holds with probability one. 
Proposition 5. The game allows for two kinds of long run equilibria:

1)Local firm monopoly: $p=F^{*}-t / 2$ and $x_{a}=\frac{1}{2}$ if and only if $\frac{3}{2} \leq \frac{F^{*}}{t}$

2) Type-I duopoly: $p=\frac{F^{*}(1+\sqrt{2})+t(3-\sqrt{2})}{4 \sqrt{2}+6}, x_{a}=\frac{2 \frac{F^{*}}{t}-1+\frac{3}{\sqrt{2}}}{3 \sqrt{2}+4}$ and $p^{*}=\frac{t(3+3 \sqrt{2})-F^{*}(\sqrt{2}+2)}{3+2 \sqrt{2}}$ if and only if $0 \leq \frac{F^{*}}{t} \leq \frac{3}{2}$

Proposition 5 allows us to provide insights on the geographical impacts of the Internet revolution in the long run. First, the type-II duopoly is never a long run equilibrium. Indeed, according to the value of $F^{*} / t$ we can have either a local firm monopoly or a type-I duopoly. Thus the prediction that "old economy" firms are bound to serve a downtown market does not seem to be supported by the maximization of (expected long run) profits in our model. The local firm will always serve the outskirts (either left or right side of the city). This result gives credit to the "delocalization" scenario (see Kotkin (2001)). The model forecasts that when the on-line firm enters the market, the local firm will ultimately leave the city center instead of keeping its downtown customers. The mechanism we describe is a purely competitive one. Note however that other arguments can be put forward to explain such behavior. The level of real-estate prices in city centers may also play an important role.

Second, the best choice for the local firm is to choose a central location to maintain the monopoly as long as possible. However, when the relative fixed $\operatorname{cost} F^{*} / t$ is low enough (more precisely $0 \leq F^{*} / t<3 / 2$, the "old economy" firm chooses to locate outside of the center of the town. As $F^{*} / t$ decreases the optimal location moves leftward.

Thus some long-run geographical impacts of the Internet may be supported by our model (namely that considered in Kotkin (2001), although his arguments differ from ours). The intuition for this result is that local monopoly effects play a leading role in Hotelling-type models. Thus, as long as the local firm wants to keep a local monopoly, it is better off not having the market share "shrink at both edges" (as would be the case in the typeII duopoly). However, when the on-line firm can enter the market, the local firm is better off accommodating this entry. More precisely, the local firm lets the on-line firm serve the far right customers, in order to keep the more profitable left side of the city.

Remark that choosing a location to sustain a type-I duopoly when blockaded entry is no longer possible avoids deterring entry. Indeed, if the local firm chooses a location to maintain the monopoly when $F^{*} / t<3 / 2$, this will be costly, since entry then has to be deterred. Moreover, there is 
no way to go from blockaded entry monopoly to type-II duopoly without a deterred entry episode (as $F^{*} / t$ decreases). This may also explain why type-II duopoly is never an equilibrium.

It is worth noting that our paper is framed in terms of geographical locations. A potential generalization of the model is that the location could also be interpreted as a measure of preferences in a horizontal differentiation model, in which downtown location corresponds to firms targeting the mainstream, while moving to the outskirts corresponds to targeting more specific segments of the population. In that light, one result of long term analysis is that old economy firms will move away from the mainstream and toward more specific markets as time goes by and $F^{*}$ falls. ${ }^{8}$

\section{Conclusion}

This article examines the competition between two firms: a local and an online firm. The local firm's customers bear a linear transportation cost while on-line customers bear a fixed cost. We argue that part of this disadvantage may come from consumers wariness of on-line transactions.

We first study short run equilibria in which the local firm's address is given. We show that the model copes with three current stylized facts. First, competitive allocations typically leave the "new economy" firm with smaller market shares. Second, this happens even though this firm offers attractive prices. Third, the profits of the on-line firm are generally lower. Our model explains these facts by a comparative disadvantage on the demand side for the on-line firm. These results are valid when on-line technology allows variable costs to be reduced. In particular, we show that the argument for a hypothetical on-line firm monopoly is that cost differences more than offset the comparative disadvantage on the demand side. This also suggests that the stylized facts of the new economy recession are more likely to stem from a net disadvantage on the demand side.

In the long run, we show that it is never advantageous for the local firm to serve a market close to the city center. On the contrary, it will tend to leave the center and force the on-line firm to serve far right customers. This allows the local firm to keep a large market share. We argue that this effect may be related to some of the predictions concerning the geographical impact of the development of remote access services (Kotkin, 2001).

It can be argued that the economic difficulties faced by the on-line firm in our model stem from the competitive allocation. It would be interesting to investigate other market structures (such as an integrated firm) ${ }^{9}$.

8 We owe this remark to an anonymous referee.

9 Details are available upon request. 
It is easy to show that an integrated firm will implement the first best solution from the social optimum viewpoint. Moreover, the socially optimal market share of the on-line firm is typically smaller than its competitive counterpart. In any case, if we look at current market sharing, it seems hard to justify subsidizing remote access technology. Note however that most of the current public support for remote access technologies points to consumer wariness. From this viewpoint, our findings agree with some of the recent requirements of the European Commission (2000). Indeed, this can be interpreted as an attempt to lower $F^{*}$, which is socially desirable if not too costly.

\section{References}

[1] d'Aspremont C., Gabszewicz J.J. and J. Thisse (1979), "On Hotelling's "Stability in Competition", Econometrica, 47, pp 1145-1150.

[2] Allen, F.A. and A.M. Santomero (2001) "What do Financial Intermediaries do ?",Journal of Banking and Finance ,21, pp. 271-294.

[3] Bain, J.S. (1956), Barriers to new competition, Harvard University Press, Cambridge, MA.

[4] Balsubramanian, S. (1998), "Mail versus Mall: A strategic Analysis of Competition Between Direct Marketers and Conventional Retailers", Marketing Science, 17, pp. 181-195.

[5] Bouckaert, J. (2000), "Monopolistic Competition with a Mail Order Business", Economics Letters , 66, pp.303-310.

[6] Bouckaert, J. and H. Degryse (1995), "Phonebanking", European Economic Review, 39, pp. 229-244.

[7] Brousseau, E. (2000), "Commerce électronique : ce que disent les chiffres et ce qu'il faudrait savoir", Economie et Statistique, 339-340, pp. 147-170.

[8] Degryse, H. (1996), "On the Interaction Between Vertical and Horizontal Product Differentiation: An Application to banking", The Journal of Industrial Economics, 44, pp. 169-185.

[9] European Commission (2000), "Commission proposal for the European Parliament and council directives on the taking up, the pursuit and the prudential supervision of the electronic money institutions", http: \\europa.eu.int/comm/internal_market/en/finances/general/1085 en.pdf.

[10] Curien, N. and K. Oubejja (1999), "Réseau multiservices : équilibres et dynamique", Annales d'Économie et de statistique, 53, pp. 214-228.

[11] Economides, N. (1996), "The Economics of Networks", International Journal of Industrial Organization, 14, pp. 673-699. 
[12] Glicksberg, I. L. (1952), "A Further Generalization of the Kakutani Fixed Point Theorem with Application to Nash Equilibrium Points", Proceedings of the American Mathematical Society, 38, pp. 170-174.

[13] Hotelling, H. (1929), "Stability in Competition", Economic Journal, 39, pp. $41-57$.

[14] Kotkin J. (2001) The New Geography: How the Digital Revolution is Reshaping the American Landscape, Random House, New York.

[15] La lettre du E-Marketing, http: \\www.lalettreduemarketing.com.

[16] Matutes, C. and A.J. Padilla (1994), "Shared ATM Networks and Banking Competition", European Economic Review, 38, pp. 1057-1069.

[17] Osborne, M. J. and C. Pitchik (1987), "Equilibrium in Hotelling's Model of Spatial Competition", Econometrica, 55, pp. 911-922.

\section{Local firm best response}

When $p^{*}+F^{*}-t x_{a} \geq p \geq p^{*}+F^{*}-t\left(1-x_{a}\right)$ the profit function of the local firm is $p\left(x_{a}+\frac{p^{*}-p+F^{*}}{t}\right)$ We have

$$
\frac{\partial}{\partial p}\left\{p\left(x_{a}+\frac{p^{*}-p+F^{*}}{t}\right)\right\}=0 \Leftrightarrow p=\frac{p^{*}+F^{*}+t x_{a}}{2}
$$

Now the condition $p^{*}+F^{*}-t x_{a} \geq \frac{p^{*}+F^{*}+t x_{a}}{2} \geq p^{*}+F^{*}-t\left(1-x_{a}\right)$ is equivalent to $-F^{*}+t\left(2-x_{a}\right) \geq p^{*} \geq-F^{*}+3 t x_{a}$. We also deduce that whenever $p^{*}+F^{*}-t x_{a} \geq p \geq p^{*}+F^{*}-t\left(1-x_{a}\right)$

$$
-F^{*}+t\left(2-x_{a}\right)<p^{*} \Rightarrow \frac{\partial}{\partial p}\left\{p\left(x_{a}+\frac{p^{*}-p+F^{*}}{t}\right)\right\}<0,
$$

and

$$
-F^{*}+3 t x_{a}>p^{*} \Rightarrow \frac{\partial}{\partial p}\left\{p\left(x_{a}+\frac{p^{*}-p+F^{*}}{t}\right)\right\}>0 .
$$

When $p^{*}+F^{*} \geq p \geq p^{*}+F^{*}-t x_{a}$, the profit function of the local firm is $2 p \frac{p^{*}-p+F^{*}}{t}$. We have

$$
\frac{\partial}{\partial p}\left\{2 p\left(\frac{p^{*}-p+F^{*}}{t}\right)\right\}=0 \Leftrightarrow p=\frac{p^{*}+F^{*}}{2}
$$


The condition $p^{*}+F^{*} \geq \frac{p^{*}+F^{*}}{2} \geq p^{*}+F^{*}-t x_{a}$, is equivalent to $-F^{*}+2 t x_{a} \geq p^{*} \geq-F^{*}$. We also deduce that whenever $p^{*}+F^{*} \geq p \geq p^{*}+$ $F^{*}-t x_{a}$

$$
-F^{*}+2 t x_{a}<p^{*} \Rightarrow \frac{\partial}{\partial p}\left\{2 p \frac{p^{*}-p+F^{*}}{t}\right\}<0,
$$

and

$$
-F^{*}>p^{*} \Rightarrow \frac{\partial}{\partial p}\left\{2 p \frac{p^{*}-p+F^{*}}{t}\right\}>0 .
$$

Now notice that $-F^{*}+2 t x_{a} \leq-F^{*}+3 t x_{a}$. Hence the conditions $-F^{*}+t\left(2-x_{a}\right) \geq p^{*} \geq-F^{*}+3 t x_{a}$ and $-F^{*}+2 t x_{a} \geq p^{*} \geq-F^{*}$ can never be met at the same time unless $x_{a}=0$. In this last case, it is trivially seen that both local maxima $\frac{p^{*}+F^{*}}{2}$ and $\frac{p^{*}+F^{*}+t x_{a}}{2}$ coincides. We thus complete the study of the maximization of the profit function of the local firm. Notice that when $-F^{*}+2 t x_{a} \leq p^{*} \leq-F^{*}+3 t x_{a}$ the maximum of the profit is reached for $p=p^{*}+F^{*}-t x_{a}$ and that the profit function is not differentiable at this point.

\section{On-line firm best response}

When $p-F^{*}+t\left(1-x_{a}\right) \geq p^{*} \geq p-F^{*}+t x_{a}$, the profit function of the on-line firm is $p^{*}\left(1-x_{a}-\frac{p^{*}-p+F^{*}}{t}\right)$. We have

$$
\frac{\partial}{\partial p^{*}}\left\{p^{*}\left(1-x_{a}-\frac{p^{*}-p+F^{*}}{t}\right)\right\}=0 \Leftrightarrow p^{*}=\frac{p-F^{*}+t\left(1-x_{a}\right)}{2} .
$$

The condition $p-F^{*}+t\left(1-x_{a}\right) \geq \frac{p-F^{*}+t\left(1-x_{a}\right)}{2} \geq p-F^{*}+t x_{a}$, is equivalent to $F^{*}-t\left(3 x_{a}-1\right) \geq p \geq F^{*}-t\left(1-x_{a}\right)$. We also deduce that whenever $p-F^{*}+t\left(1-x_{a}\right) \geq p^{*} \geq p-F^{*}+t x_{a}$,

$$
F^{*}-t\left(3 x_{a}-1\right)<p \Rightarrow \frac{\partial}{\partial p^{*}}\left\{p^{*}\left(1-x_{a}-\frac{p^{*}-p+F^{*}}{t}\right)\right\}<0
$$

and

$$
F^{*}-t\left(1-x_{a}\right)>p \Rightarrow \frac{\partial}{\partial p^{*}}\left\{p^{*}\left(1-x_{a}-\frac{p^{*}-p+F^{*}}{t}\right)\right\}>0
$$


When $p-F^{*}+t x_{a} \geq p^{*} \geq p \geq p-F^{*}$, the profit function of the on-line firm is $p^{*}\left(1-2 \frac{p^{*}-p+F^{*}}{t}\right)$. We have

$$
\frac{\partial}{\partial p^{*}}\left\{p^{*}\left(1-2 \frac{p^{*}-p+F^{*}}{t}\right)\right\}=0 \Leftrightarrow p^{*}=\frac{p-F^{*}+t / 2}{2} .
$$

The condition $p-F^{*}+t x_{a} \geq \frac{p-F^{*}+t / 2}{2} \geq p-F^{*}$ is equivalent to $F^{*}+t / 2 \geq$ $p \geq F^{*}-t\left(2 x_{a}-1 / 2\right)$. Moreover we deduce that whenever $p-F^{*}+t x_{a} \geq$ $p^{*} \geq p-F^{*}$

$$
F^{*}+t / 2<p \Rightarrow \frac{\partial}{\partial p^{*}}\left\{p^{*}\left(1-2 \frac{p^{*}-p+F^{*}}{t}\right)\right\}<0,
$$

and

$$
F^{*}-t\left(2 x_{a}-1 / 2\right)>p \Rightarrow \frac{\partial}{\partial p^{*}}\left\{p^{*}\left(1-2 \frac{p^{*}-p+F^{*}}{t}\right)\right\}>0 .
$$

Contrarily to the case of the local firm, the two conditions $F^{*}-t\left(2 x_{a}-1 / 2\right) \leq p \leq F^{*}+t / 2$ and $F^{*}-t\left(1-x_{a}\right) \leq p \leq F^{*}-t\left(3 x_{a}-1\right)$ may both be met for some values of $p$. Thus local maxima must be compared.

First, notice that we always have $F^{*}-t\left(3 x_{a}-1\right) \geq F^{*}-t\left(2 x_{a}-1 / 2\right)$ as this inequality is equivalent to $x_{a} \leq 1 / 2$. Also $F^{*}-t\left(1-x_{a}\right) \leq F^{*}-$ $t\left(2 x_{a}-1 / 2\right)$ is equivalent to $x_{a} \leq 1 / 2$. But $F^{*}-t\left(3 x_{a}-1\right) \leq F^{*}+t / 2$ is equivalent to $x_{a} \geq 1 / 6$. We then distinguish two cases.

\subsection{Case $x_{a} \leq 1 / 6$}

For these values of $x_{a}$ we have $F^{*}-t\left(3 x_{a}-1\right)>F^{*}-t\left(2 x_{a}-1 / 2\right) \geq$ $F^{*}+t / 2>F^{*}-t\left(1-x_{a}\right)$. First, if $p>F^{*}-t\left(3 x_{a}-1\right)$ we have, over the relevant intervals,

$$
\begin{aligned}
& \frac{\partial}{\partial p^{*}}\left\{p^{*}\left(1-2 \frac{p^{*}-p+F^{*}}{t}\right)\right\}<0 \\
& \frac{\partial}{\partial p^{*}}\left\{p^{*}\left(1-x_{a}-\frac{p^{*}-p+F^{*}}{t}\right)\right\}<0
\end{aligned}
$$

and the maximum of the profit is then reached when $p^{*}=p-F^{*}$.

Now if $F^{*}-t\left(3 x_{a}-1\right) \leq p \leq F^{*}-t\left(2 x_{a}-1 / 2\right)$, the shape of the profit function is as follows: when $p^{*} \leq p-F^{*}$, the profit increases linearly with $p^{*}$; if $p+-F^{*} \leq p^{*} \leq p-F^{*}+t x_{a}$, the profit decreases as a quadratic function of $p^{*}$; if $p-F^{*}+t x_{a} \leq p^{*} \leq p-F^{*}+t\left(1-x_{a}\right)$ the profit increases then reached a local maximum for $p^{*}=\frac{p-F^{*}+t\left(1-x_{a}\right)}{2}$; finally if 
$p>p-F^{*}+t\left(1-x_{a}\right)$, the profit always decreases with $p^{*}$. We then have to compare the local maxima $p^{*}=p-F^{*}$ and $p^{*}=\frac{p-F^{*}+t\left(1-x_{a}\right)}{2}$. We get

$$
\begin{aligned}
& \pi^{*}\left(x_{a}, p, p-F^{*}\right)=p-F^{*} \\
& \pi^{*}\left(x_{a}, p,\left(p-F^{*}+t\left(1-x_{a}\right)\right) / 2\right)=\frac{\left(p-F^{*}+t\left(1-x_{a}\right)\right)^{2}}{4 t}
\end{aligned}
$$

Now one easily shows that when $p \leq F^{*}-t\left(3 x_{a}-1\right)$ the condition $\frac{\left(p-F^{*}+t\left(1-x_{a}\right)\right)^{2}}{4 t} \geq p-F^{*}$ is equivalent to $p \leq F^{*}+t\left(1-\sqrt{x_{a}}\right)^{2}$.

We then have to study the conditions under which $F^{*}-t\left(3 x_{a}-1\right) \geq$ $F^{*}+t\left(1-\sqrt{x_{a}}\right)^{2} \geq F^{*}+t / 2$. The condition $F^{*}-t\left(3 x_{a}-1\right) \geq F^{*}+t\left(1-\sqrt{x_{a}}\right)^{2}$ is equivalent to $2 \sqrt{x_{a}}\left(-1+2 \sqrt{x_{a}}\right) \leq 0$, which always holds if $x_{a}<1 / 6$. However, the condition $F^{*}+t\left(1-\sqrt{x_{a}}\right)^{2} \geq F^{*}+t / 2$ is equivalent to $x_{a} \leq 3 / 2-$ $\sqrt{2}<1 / 6$. Two subcases must then be distinguished.

If $0 \leq x_{a} \leq 3 / 2-\sqrt{2}$ then $\pi^{*}\left(x_{a}, p, p-F^{*}\right) \geq \pi^{*}\left(x_{a}, p,\left(p-F^{*}+t\left(1-x_{a}\right)\right) / 2\right)$ if $F^{*}-t\left(3 x_{a}-1\right) \geq p \geq F^{*}+t\left(1-\sqrt{x_{a}}\right)^{2}$. The maximum of the profit is then reached when $p^{*}=p-F^{*}$. If $F^{*}+t\left(1-\sqrt{x_{a}}\right)^{2} \geq p \geq F^{*}+t / 2$ then maximum of the profit is reached at price $p^{*}=\left(p-F^{*}+t\left(1-x_{a}\right)\right) / 2$.

If $3 / 2-\sqrt{2} \leq x_{a} \leq 1 / 6$, the maximum of the profit is always reached at price $p^{*}=p-F^{*}$ when $F^{*}-t\left(3 x_{a}-1\right) \geq p \geq F^{*}+t / 2$.

Consider now the case $F^{*}+t / 2 \geq p \geq F^{*}-t\left(2 x_{a}-1 / 2\right)$. In this case we have to compare the two local maxima

$$
\begin{aligned}
& \pi^{*}\left(x_{a}, p,\left(p-F^{*}+t / 2\right) / 2\right)=\frac{\left(p-F^{*}+t / 2\right)^{2}}{2 t} \\
& \pi^{*}\left(x_{a}, p,\left(p-F^{*}+t\left(1-x_{a}\right)\right) / 2\right)=\frac{\left(p-F^{*}+t\left(1-x_{a}\right)\right)^{2}}{4 t}
\end{aligned}
$$

It is not difficult to show that if $p \geq F^{*}-t\left(2 x_{a}-1 / 2\right)$, we have $\pi^{*}\left(x_{a}, p,\left(p-F^{*}+t / 2\right) / 2\right) \leq \pi^{*}\left(x_{a}, p,\left(p-F^{*}+t\left(1-x_{a}\right)\right) / 2\right)$ if and only if $p \leq F^{*}-t\left((\sqrt{2}+1) x_{a}-1 / \sqrt{2}\right)$.

Now we always have $\left.F^{*}-t\left((\sqrt{2}+1) x_{a}-1 / \sqrt{2}\right) \geq F^{*}-t\left(2 x_{a}-1 / 2\right)\right)$. However, $F^{*}-t\left((\sqrt{2}+1) x_{a}-1 / \sqrt{2}\right) \leq F^{*}+t / 2$ is equivalent to $x_{a} \geq 3 / 2-\sqrt{2}$, and two subcases must again be distinguished. First, if $x_{a} \leq 3 / 2-\sqrt{2}$ the maximum of the profit is reached at price $p^{*}=\left(p-F^{*}+t\left(1-x_{a}\right)\right) / 2$ whenever $F^{*}+t / 2 \geq p \geq F^{*}-t\left(2 x_{a}-1 / 2\right)$. Second, if $3 / 2-\sqrt{2} \leq x_{a} \leq 1 / 6$, the maximum of the profit is reached at price $p^{*}=\left(p-F^{*}+t / 2\right) / 2$ if $F^{*}+t / 2 \geq$ 
$p \geq F^{*}-t\left((\sqrt{2}+1) x_{a}-1 / \sqrt{2}\right)$, and at price $p^{*}=\left(p-F^{*}+t\left(1-x_{a}\right)\right) / 2$ whenever $\left.F^{*}-t\left((\sqrt{2}+1) x_{a}-1 / \sqrt{2}\right) \geq p \geq F^{*}-t\left(2 x_{a}-1 / 2\right)\right)$.

Now consider the case $F^{*}-t\left(1-x_{a}\right) \leq p \leq F^{*}-t\left(2 x_{a}-1 / 2\right)$. Clearly, in this case, the maximum of the profit is reached at price $p-F^{*}+t\left(1-x_{a}\right)$.

Finally, if $p<F^{*}-t\left(1-x_{a}\right)$, it is optimal not to produce, that is $p^{*}$ can take any value larger than $p-F^{*}+t\left(1-x_{a}\right)$.

\subsection{Case $x_{a}>1 / 6$}

If $x_{a}>1 / 6$, we now have the following ordering $F^{*}+t / 2>F^{*}-t\left(3 x_{a}-1\right) \geq$ $F^{*}-t\left(2 x_{a}-1 / 2\right) \geq F^{*}-t\left(1-x_{a}\right)$.

The first case to be considered is then $p>F^{*}+t / 2$. In this case, the maximum of the profit is reached at price $p^{*}=p-F^{*}$.

Next, if $F^{*}+t / 2 \geq p \geq F^{*}-t\left(3 x_{a}-1\right)$, the maximum of the profit is reached at price $p^{*}=\left(p-F^{*}+t / 2\right) / 2$.

If $F^{*}-t\left(3 x_{a}-1\right) \geq p \geq F^{*}-t\left(2 x_{a}-1 / 2\right)$, we have two local maxima for $p^{*}=\left(p-F^{*}+t / 2\right) / 2$ and $p^{*}=\left(p-F^{*}+t\left(1-x_{a}\right)\right) / 2$. We already mentioned that if $p \geq F^{*}-t\left(2 x_{a}-1 / 2\right)$ we have $\pi^{*}\left(x_{a}, p,\left(p-F^{*}+t / 2\right) / 2\right) \leq \pi^{*}\left(x_{a}, p\right.$, $\left.\left(p-F^{*}+t\left(1-x_{a}\right)\right) / 2\right)$ if and only if $p \leq F^{*}-t\left((\sqrt{2}+1) x_{a}-1 / \sqrt{2}\right)$. We also know that $F^{*}-t\left((\sqrt{2}+1) x_{a}-1 / \sqrt{2}\right) \geq F^{*}-t\left(2 x_{a}-1 / 2\right)$. The only new point we have to check is whether $F^{*}-t\left((\sqrt{2}+1) x_{a}-1 / \sqrt{2}\right) \leq F^{*}-t\left(3 x_{a}-1\right)$. This inequality is equivalent to $x_{a} \leq 1 / 2$. Thus we always have $F^{*}-t\left(2 x_{a}-\right.$ $1 / 2) \leq F^{*}-t\left((\sqrt{2}+1) x_{a}-1 / \sqrt{2}\right) \leq F^{*}-t\left(3 x_{a}-1\right)$ and we deduce that the maximum of the profit is reached at price $p^{*}=\left(p-F^{*}+t / 2\right) / 2$ if $F^{*}-t$ $\left((\sqrt{2}+1) x_{a}-1 / \sqrt{2}\right) \leq p \leq F^{*}-t\left(3 x_{a}-1\right)$ and at price $p^{*}=\left(p-F^{*}+t(1-\right.$ $\left.\left.x_{a}\right)\right) / 2$ if $F^{*}-t\left(2 x_{a}-1 / 2\right) \leq p \leq F^{*}-t\left((\sqrt{2}+1) x_{a}-1 / \sqrt{2}\right)$.

The next two cases are easy. If $F^{*}-t\left(2 x_{a}-1 / 2\right) \geq p \geq F^{*}-t\left(1-x_{a}\right)$ the profit is maximum at price $p^{*}=\left(p-F^{*}+t\left(1-x_{a}\right)\right) / 2$. Finally if $p<F^{*}-t\left(1-x_{a}\right)$, it is again optimal not to produce that is to choose $p^{*} \geq p-F^{*}+t\left(1-x_{a}\right)$.

\section{Computation of pure strategy equilibria}

Our computation of the equilibria relies on a "brute force" approach. We simply consider the best response functions of both firms and compute the intersections. 
Let us recall the best response functions

- local firm

$$
\begin{array}{llrl}
p & \geq p^{*}+F^{*} & \text { if } & p^{*} \leq-F^{*} \\
p & =\frac{p^{*}+F^{*}}{2} & & \text { if } \quad-F^{*} \leq p^{*} \leq-F^{*}+2 t x_{a} \\
p=p^{*}+F^{*}-t x_{a} & \text { if } \quad-F^{*}+2 t x_{a} \leq p^{*} \leq-F^{*}+3 t x_{a} \\
p=\frac{p^{*}+F^{*}+t x_{a}}{2} & \text { if } \quad-F^{*}+3 t x_{a} \leq p^{*} \leq-F^{*}+t\left(2-x_{a}\right) \\
p=p^{*}+F^{*}-t\left(1-x_{a}\right) & \text { if } \quad-F^{*}+t\left(2-x_{a}\right) \leq p^{*}
\end{array}
$$

- on-line firm (case $\left.0 \leq x_{a} \leq \frac{3}{2}-\sqrt{2}\right)$

$$
\begin{array}{lll}
p^{*} \geq p-F^{*}+t\left(1-x_{a}\right) & \text { if } & p \leq F^{*}-t\left(1-x_{a}\right) \\
p^{*}=\frac{p-F^{*}+t\left(1-x_{a}\right)}{2} & \text { if } & F^{*}-t\left(1-x_{a}\right) \leq p \leq F^{*}+t\left(1-\sqrt{x_{a}}\right)^{2} \\
p^{*}=p-F^{*} & \text { if } & p \geq F^{*}+t\left(1-\sqrt{x_{a}}\right)^{2}
\end{array}
$$

- on-line firm (case $\left.\frac{3}{2}-\sqrt{2} \leq x_{a} \leq \frac{1}{2}\right)$

$$
\begin{array}{lll}
p^{*} \geq p-F^{*}+t\left(1-x_{a}\right) & \text { if } & p \leq F^{*}-t\left(1-x_{a}\right) \\
p^{*}=\frac{p-F^{*}+t\left(1-x_{a}\right)}{2} & \text { if } & F^{*}-t\left(1-x_{a}\right) \leq p \leq F^{*}-t\left((\sqrt{2}+1) x_{a}-\frac{1}{\sqrt{2}}\right) \\
p^{*}=\frac{p-F^{*}+t / 2}{2} & \text { if } & F^{*}-t\left((\sqrt{2}+1) x_{a}-\frac{1}{\sqrt{2}}\right) \leq p \leq F^{*}+\frac{t}{2} \\
p^{*}=p-F^{*} & \text { if } & p \geq F^{*}+\frac{t}{2}
\end{array}
$$

As the complete derivation is quite cumbersome, and in an attempt to save time for the careful and courageous reader, we always adopt the same redaction.

Each intersection of the best responses is the solution of a two(in)equations linear system. For each case we first compute the solutions of this system. Second, we derive the conditions under which these solutions of the linear system belong to the relevant intervals. Third, we derive the conditions under which the solutions of the linear provide positive prices.

\subsection{Case (a)}

This case would correspond to a situation in which $\Delta=0$. The conditions under which the on-line firm can monopolize the market correspond to the case $F^{*}<0$ which is not considered in the paper.

\subsection{Case (b)}

This case corresponds to situations under which the best response function of the local firm does not depend on $x_{a}$. 


\subsubsection{Cases (b) and (a')}

$$
\begin{aligned}
& p=\frac{p^{*}+F^{*}}{2} \\
& p^{*} \geq p-F^{*}+t\left(1-x_{a}\right)
\end{aligned}
$$

Both conditions imply $p^{*} \geq \frac{p^{*}+F^{*}}{2}-F^{*}+t\left(1-x_{a}\right)$ or $p^{*} \geq-F^{*}+$ $2 t\left(1-x_{a}\right)$. Now the relevant interval conditions for (b) are $-F^{*} \leq p^{*} \leq$ $-F^{*}+2 t x_{a}$. As $-F^{*}+2 t\left(1-x_{a}\right) \leq-F^{*}+2 t x_{a}$ implies $x_{a}=1 / 2$, we have $p^{*}=-F^{*}+t$. Now this induces $p=t / 2$.

The positivity requirement for $p^{*}$ implies $F^{*} / t \leq 1$. Now the relevant interval conditions for (a') are $1 \leq F^{*} / t$, hence $F^{*} / t=1$, and $p^{*}=0$.

We finally get the following set of conditions

$$
\begin{aligned}
& p=t / 2 \\
& p^{*}=0 \\
& x_{a}=1 / 2 \\
& F^{*} / t=1
\end{aligned}
$$

Under this case the profits are $\pi=t / 2, \pi^{*}=0$.

\subsubsection{Cases (b) and (b')}

$$
\begin{aligned}
& p=\frac{p^{*}+F^{*}}{2} \\
& p^{*}=\frac{p-F^{*}+t\left(1-x_{a}\right)}{2}
\end{aligned}
$$

The conditions are equivalent to

$$
\begin{aligned}
& p=\frac{F^{*}+t\left(1-x_{a}\right)}{3} \\
& p^{*}=\frac{2 t\left(1-x_{a}\right)-F^{*}}{3}
\end{aligned}
$$

If $0 \leq x_{a} \leq 3 / 2-\sqrt{2}$ the relevant interval conditions are

$$
\begin{aligned}
& F^{*}-t\left(1-x_{a}\right) \leq \frac{F^{*}+t\left(1-x_{a}\right)}{3} \leq F^{*}+t\left(1-\sqrt{x_{a}}\right)^{2} \\
& -F^{*} \leq \frac{2 t\left(1-x_{a}\right)-F^{*}}{3} \leq-F^{*}+2 t x_{a}
\end{aligned}
$$

This is equivalent to

$$
\begin{aligned}
& \left(1-x_{a}\right) / 2-\frac{3}{2}\left(1-\sqrt{x_{a}}\right)^{2} \leq F^{*} / t \leq 2\left(1-x_{a}\right) \\
& -1+x_{a} \leq F^{*} / t \leq-1+4 x_{a}
\end{aligned}
$$

But this implies in particular $-1+4 x_{a} \geq\left(1-x_{a}\right) / 2-\frac{3}{2}\left(1-\sqrt{x_{a}}\right)^{2}$ or $0 \geq-6 x_{a}+3 \sqrt{x_{a}}$, which implies $x_{a}=0$ and $F^{*} / t=-1$, which is not possible since $F^{*} \quad 0$. 
Now if $3 / 2-\sqrt{2} \leq x_{a} \leq 1 / 2$ the relevant interval conditions are

$$
\begin{aligned}
& F^{*}-t\left(1-x_{a}\right) \leq \frac{F^{*}+t\left(1-x_{a}\right)}{3} \leq F^{*}-t\left((\sqrt{2}+1) x_{a}-\frac{1}{\sqrt{2}}\right) \\
& -F^{*} \leq \frac{2 t\left(1-x_{a}\right)-F^{*}}{3} \leq-F^{*}+2 t x_{a}
\end{aligned}
$$

this is equivalent to

$$
\begin{aligned}
& \left(\frac{3}{\sqrt{2}}+1\right) x_{a}-\frac{3}{2 \sqrt{2}}+\frac{1}{2} \leq F^{*} / t \leq 2\left(1-x_{a}\right) \\
& -1+x_{a} \leq F^{*} / t \leq-1+4 x_{a}
\end{aligned}
$$

Again, we must have $-1+4 x_{a} \geq\left(\frac{3}{\sqrt{2}}+1\right) x_{a}-\frac{3}{2 \sqrt{2}}+\frac{1}{2}$ which is equivalent to $x_{a} \geq 1 / 2$. Hence, we get $x_{a}=1 / 2$. In this case $\left(\frac{3}{\sqrt{2}}+1\right) x_{a}-\frac{3}{2 \sqrt{2}}+$ $\frac{1}{2} \leq F^{*} / t \leq 2\left(1-x_{a}\right)$ writes $1 \leq F^{*} / t \leq 1$ thus $F^{*} / t=1$, and this together with $x_{a}=1 / 2$ implies $p^{*}=0$ and $p=t / 2$. We are then back to (1).

\subsubsection{Cases (b) and (c')}

$$
\begin{aligned}
& p=\frac{p^{*}+F^{*}}{2} \\
& p^{*}=\frac{p-F^{*}+t / 2}{2}
\end{aligned}
$$

The system solves as

$$
\begin{aligned}
& p=\frac{F^{*}+t / 2}{3} \\
& p^{*}=\frac{t-F^{*}}{3}
\end{aligned}
$$

The relevant interval conditions are

$$
\begin{aligned}
& -F^{*} \leq \frac{t-F^{*}}{3} \leq-F^{*}+2 t x_{a} \\
& F^{*}-t\left((\sqrt{2}+1) x_{a}-\frac{1}{\sqrt{2}}\right) \leq \frac{F^{*}+t / 2}{3} \leq F^{*}+\frac{t}{2}
\end{aligned}
$$

They are equivalent to

$$
\begin{aligned}
& -\frac{1}{2} \leq F^{*} / t \leq-1 / 2+3 x_{a} \\
& -\frac{1}{2} \leq F^{*} / t \leq \frac{1}{4}-\frac{3}{2 \sqrt{2}}+\frac{3(\sqrt{2}+1) x_{a}}{2}
\end{aligned}
$$

We check that $\frac{1}{4}-\frac{3}{2 \sqrt{2}}+\frac{3(\sqrt{2}+1) x_{a}}{2} \geq-\frac{1}{2}$ is equivalent to $x_{a} \geq \frac{3}{2}-\sqrt{2}$ which always holds as (c') requires this condition.

Also we have that $-1 / 2+3 x_{a} \geq \frac{1}{4}-\frac{3}{2 \sqrt{2}}+\frac{3(\sqrt{2}+1) x_{a}}{2}$ is equivalent to $x_{a} \leq \frac{1}{2}$. Hence the relevant interval conditions simplify to $-\frac{1}{2} \leq F^{*} / t \leq \frac{1}{4}-$ $\frac{3}{2 \sqrt{2}}+\frac{3(\sqrt{2}+1) x_{a}}{2}$. 
The positivity requirement for $p$ writes $F^{*} / t \geq-\frac{1}{2}$ and that for $p^{*}$ leads to $F^{*} / t \leq 1$, conditions which are weaker than the above relevant interval conditions. We thus find a new intersection

$$
\begin{aligned}
& p=\frac{F^{*}+t / 2}{3} \\
& p^{*}=\frac{t-F^{*}}{3} \\
& 0 \leq F^{*} / t \leq \frac{1}{4}-\frac{3}{2 \sqrt{2}}+\frac{3(\sqrt{2}+1) x_{a}}{2}
\end{aligned}
$$

(The condition $x_{a} \geq \frac{3}{2}-\sqrt{2}$ is included in $-\frac{1}{2} \leq \frac{1}{4}-\frac{3}{2 \sqrt{2}}+\frac{3(\sqrt{2}+1) x_{a}}{2}$.) Under these conditions we have $\pi=2 t\left(\frac{F^{*} / t+1 / 2}{3}\right)^{2}$ and $\pi^{*}=2 t\left(\frac{1-F^{*} / t}{3}\right)^{2}$. Notice finally that this solution encompasses (1).

\subsubsection{Cases (b) and (d')}

$$
\begin{aligned}
& p=\frac{p^{*}+F^{*}}{2} \\
& p^{*}=p-F^{*}
\end{aligned}
$$

This gives

$$
\begin{aligned}
& p=0 \\
& p^{*}=-F^{*}
\end{aligned}
$$

The relevant interval conditions for (b) give $-F^{*} \leq-F^{*} \leq-F^{*}+2 t x_{a}$ which is trivially satisfied. The relevant interval conditions for (d') when $0 \leq x_{a} \leq \frac{3}{2}-\sqrt{2}$ write $0 \geq F^{*}+t\left(1-\sqrt{x_{a}}\right)^{2}$ or $F^{*} / t \leq-\left(1-\sqrt{x_{a}}\right)^{2}$ which is again a particular case of $(1)$.

Now when $\frac{3}{2}-\sqrt{2} \leq x_{a} \leq \frac{1}{2}$ the relevant interval conditions are $0 \geq F^{*}+\frac{1}{2}$ and again we are back to $(2)$.

\section{$3.3 \quad$ Case (c)}

This case correspond to situations in which the first order condition for the maximization of $\pi$ are not fulfilled.

\subsubsection{Cases (c) and (a')}

$$
\begin{aligned}
& p=p^{*}+F^{*}-t x_{a} \\
& p^{*} \geq p-F^{*}+t\left(1-x_{a}\right)
\end{aligned}
$$


This implies $p^{*} \geq p^{*}+F^{*}-t x_{a}-F^{*}+t\left(1-x_{a}\right)$ or $0 \geq t$ which is impossible.

\subsubsection{Cases (c) and (b')}

$$
\begin{aligned}
& p=p^{*}+F^{*}-t x_{a} \\
& p^{*}=\frac{p-F^{*}+t\left(1-x_{a}\right)}{2}
\end{aligned}
$$

This gives

$$
\begin{aligned}
& p=F^{*}+t\left(1-3 x_{a}\right) \\
& p^{*}=t\left(1-2 x_{a}\right)
\end{aligned}
$$

The relevant interval conditions for (c) write $-F^{*}+2 t x_{a} \leq t\left(1-2 x_{a}\right)$ $\leq-F^{*}+3 t x_{a}$. It is equivalent to $-1+4 x_{a} \leq F^{*} / t \leq-1+5 x_{a}$. If $0 \leq x_{a} \leq$ $\frac{3}{2}-\sqrt{2}$ the relevant interval conditions for (b') write $F^{*}-t\left(1-x_{a}\right) \leq F^{*}+$ $t\left(1-3 x_{a}\right) \leq F^{*}+t\left(1-\sqrt{x_{a}}\right)^{2}$. This is equivalent to $1 / 4 \leq x_{a} \leq 1 / 2$. But $1 / 4>\frac{3}{2}-\sqrt{2}$ thus this condition is never fulfilled.

Now if $\frac{3}{2}-\sqrt{2} \leq x_{a} \leq \frac{1}{2}$ the relevant interval conditions for (b') write $F^{*}-t\left(1-x_{a}\right) \leq F^{*}+t\left(1-3 x_{a}\right) \leq F^{*}-t\left((\sqrt{2}+1) x_{a}-\frac{1}{\sqrt{2}}\right)$. It is equivalent to $x_{a}=1 / 2$. In this case we then have $p^{*}=0$ and $p=F^{*}-t / 2$. The positivity requirement for $p$ implies $F^{*} / t \geq 1 / 2$, but this statement is included in the condition $-1+4 x_{a} \leq F^{*} / t \leq 1+5 x_{a}$ when $x_{a}=1 / 2$.

We then derived the following condition

$$
\begin{aligned}
& p=F^{*}-t / 2 \\
& p^{*}=0 \\
& 1 \leq F^{*} / t \leq 7 / 2 \\
& x_{a}=1 / 2
\end{aligned}
$$

Under these conditions the profits are $\pi=F^{*}-t / 2$ and $\pi^{*}=0$.

\subsubsection{Cases (c) and (c')}

$$
\begin{aligned}
& p=p^{*}+F^{*}-t x_{a} \\
& p^{*}=\frac{p-F^{*}+t / 2}{2}
\end{aligned}
$$

This system gives the following solutions

$$
\begin{aligned}
& p=F^{*}-t\left(2 x_{a}-\frac{1}{2}\right) \\
& p^{*}=t\left(\frac{1}{2}-x_{a}\right)
\end{aligned}
$$


The relevant interval conditions for $\left(c^{\prime}\right)$ are $F^{*}-t\left((\sqrt{2}+1) x_{a}-\frac{1}{\sqrt{2}}\right)$ $\leq F^{*}-t\left(2 x_{a}-\frac{1}{2}\right) \leq F^{*}+\frac{t}{2}$. It is equivalent to $\frac{1}{2} \leq x_{a}$, we thus deduce $x_{a}-\frac{1}{2}$, $p^{*}=0$ and $p=F^{*}-t / 2$. For this values of $p^{*}$ and $x_{a}$ the relevant interval conditions for (c) imply $-F^{*}+t \leq 0 \leq-F^{*}+3 t / 2$ or $1 \leq F^{*} / t \leq 3 / 2$. We thus get a particular case of (3) above.

\subsubsection{Cases (c) and (d')}

$$
\begin{aligned}
& p=p^{*}+F^{*}-t x_{a} \\
& p^{*}=p-F^{*}
\end{aligned}
$$

This system implies $x_{a}=0$, and, as a result $p^{*}=p-F^{*}$. The relevant interval conditions for (c) then write $-F^{*} \leq p-F^{*} \leq-F^{*}$ hence $p=0$ and $p^{*}=-F^{*}$. The relevant interval conditions for (d') write $0 \geq F^{*}+t$ or $-1 \geq F^{*} / t$. Finally the positivity requirement for $p^{*}$ gives $F^{*} \leq 0$. This case is not considered here.

\subsection{Case (d)}

In this case, the local firm serves the left part of the market.

\subsubsection{Cases (d) and (a')}

$$
\begin{aligned}
& p=\frac{p^{*}+F^{*}+t x_{a}}{2} \\
& p^{*} \geq p-F^{*}+t\left(1-x_{a}\right)
\end{aligned}
$$

These conditions implies $p^{*} \geq-F^{*}+t\left(2-x_{a}\right)$. Now the relevant interval conditions for $(\mathrm{d})$ are $-F^{*}+3 t x_{a} \leq p^{*} \leq-F^{*}+t\left(2-x_{a}\right)$, hence $p^{*}=-F^{*}+t\left(2-x_{a}\right)$ and $p=t$. The relevant interval conditions for (a') write $t \leq F^{*}-t\left(1-x_{a}\right)$ or $2-x_{a} \leq F^{*} / t$. Now the positivity requirement for $p^{*}$ is $-F^{*}+t\left(2-x_{a}\right) \geq 0$ or $2-x_{a} \geq F^{*} / t$, hence $F^{*} / t=2-x_{a}$ and $p^{*}=0$. We thus reaches the following condition

$$
\begin{aligned}
& p=t \\
& p^{*}=0 \\
& F^{*} / t=2-x_{a}
\end{aligned}
$$

Under these conditions we have $\pi=t, \pi^{*}=0$. 


\subsubsection{Cases (d) and (b')}

$$
\begin{aligned}
& p=\frac{p^{*}+F^{*}+t x_{a}}{2} \\
& p^{*}=\frac{p-F^{*}+t\left(1-x_{a}\right)}{2}
\end{aligned}
$$

The solution of the system gives

$$
\begin{aligned}
& p=\frac{F^{*}+t\left(1+x_{a}\right)}{3} \\
& p^{*}=\frac{t\left(2-x_{a}\right)-F^{*}}{3}
\end{aligned}
$$

The relevant interval conditions for (d) are $-1+5 x_{a} \leq F^{*} / t \leq 2-x_{a}$.

If $0 \leq x_{a} \leq 3 / 2-\sqrt{2}$ the relevant interval conditions for ( $\left.\mathrm{b}^{\prime}\right)$ write $F^{*}-t\left(1-x_{a}\right) \leq \frac{F^{*}+t\left(1+x_{a}\right)}{3} \leq F^{*}+t\left(1-\sqrt{x_{a}}\right)^{2}$. It is equivalent to $-1-x_{a}+$ $3 \sqrt{x_{a}} \leq F^{*} / t \leq 2-x_{a}$. The positivity requirement for $p^{*}$ is $t\left(2-x_{a}\right)-F^{*} \geq 0$ or $F^{*} / t \leq 2-x_{a}$, and the positivity requirement for $p$ is $F^{*}+t\left(1+x_{a}\right) \geq 0$ or $-1-x_{a} \leq F^{*} / t$. Now when $0 \leq x_{a} \leq 3 / 2-\sqrt{2}$ we have obviously $-1-x_{a} \leq$ $-1+5 x_{a}$. Moreover $-1+5 x_{a} \leq-1-x_{a}+3 \sqrt{x_{a}}$ is equivalent $x_{a} \leq 1 / 4$ and $1 / 4>3 / 2-\sqrt{2}$. The most stringent conditions are then $-1-x_{a}+3 \sqrt{x_{a}} \leq$ $F^{*} / t \leq 2-x_{a}$.

If $3 / 2-\sqrt{2} \leq x_{a} \leq 1 / 2$ the relevant interval conditions for (b') write $F^{*}-t\left(1-x_{a}\right) \leq \frac{F^{*}+t\left(1+x_{a}\right)}{3} \leq F^{*}-t\left((\sqrt{2}+1) x_{a}-\frac{1}{\sqrt{2}}\right)$. It is equivalent to $\left(\frac{3}{\sqrt{2}}+2\right) x_{a}+\frac{1}{2}-\frac{3}{2 \sqrt{2}} \leq F^{*} / t \leq 2-x_{a}$. The positivity requirements for the prices are, as above, $-1-x_{a} \leq F^{*} / t \leq 2-x_{a}$. Now, if $3 / 2-\sqrt{2} \leq x_{a} \leq 1 / 2$ we have $-1+5 x_{a} \leq\left(\frac{3}{\sqrt{2}}+2\right) x_{a}+\frac{1}{2}-\frac{3}{2 \sqrt{2}}$, for it is equivalent to $1 / 2 \geq x_{a}$. The most stringent condition is then $\left(\frac{3}{\sqrt{2}}+2\right) x_{a}+\frac{1}{2}-\frac{3}{2 \sqrt{2}} \leq F^{*} / t \leq 2-x_{a}$.

Remark that when $3 / 2-\sqrt{2}=x_{a}$ we have $\left(\frac{3}{\sqrt{2}}+2\right) x_{a}+\frac{1}{2}-\frac{3}{2 \sqrt{2}}=$ $\frac{-\sqrt{2}+1}{2}$. On the other hand, for this value of $x_{a}$ we also have $-1-x_{a}+$ $3 \sqrt{x_{a}}=\frac{-\sqrt{2}+1}{2}$.

The conditions for this new intersection are then

$$
\begin{gathered}
p=\frac{F^{*}+t\left(1+x_{a}\right)}{3} \\
p^{*}=\frac{t\left(2-x_{a}\right)-F^{*}}{3} \\
\left(-1-x_{a}+3 \sqrt{x_{a}}\right) \mathbf{1}_{0 \leq x_{a}<3 / 2-\sqrt{2}}+\left(\left(\frac{3}{\sqrt{2}}+2\right) x_{a}+\frac{1}{2}-\frac{3}{2 \sqrt{2}}\right) 1_{3 / 2-\sqrt{2} \leq x_{a} \leq 1 / 2} \leq F^{*} / t \leq 2-x_{a}
\end{gathered}
$$


Under these conditions we have $\pi=t\left(\frac{F^{*} / t+1+x_{a}}{3}\right)^{2}$ and $\pi^{*}=t\left(\frac{2-x_{a}-F^{*} / t}{3}\right)^{2}$

\subsubsection{Cases (d) and (c')}

$$
\begin{aligned}
& p=\frac{p^{*}+F^{*}+t x_{a}}{2} \\
& p^{*}=\frac{p-F^{*}+t / 2}{2}
\end{aligned}
$$

This system admits the following solution

$$
\begin{aligned}
& p=\frac{F^{*}+t\left(1 / 2+2 x_{a}\right)}{3} \\
& p^{*}=\frac{t\left(1+x_{a}\right)-F^{*}}{3}
\end{aligned}
$$

The relevant interval conditions for $(\mathrm{d})$ are $-F^{*}+3 t x_{a} \leq \frac{t\left(1+x_{a}\right)-F^{*}}{3}$ $\leq-F^{*}+t\left(2-x_{a}\right)$. It is equivalent to $-1 / 2+4 x_{a} \leq F^{*} / t \leq 5 / 2-2 x_{a}$.

The relevant interval conditions for $\left(\mathrm{c}^{\prime}\right)$ are $F^{*}-t\left((\sqrt{2}+1) x_{a}-\frac{1}{\sqrt{2}}\right)$ $\leq \frac{F^{*}+t\left(1 / 2+2 x_{a}\right)}{3} \leq F^{*}+\frac{t}{2}$. It is equivalent to $x_{a}-1 / 2 \leq F^{*} / t \leq(3 / \sqrt{2}+$ $5 / 2) x_{a}-\frac{6-\sqrt{2}}{4 \sqrt{2}}$. Now notice $4 x_{a}-1 / 2 \leq(3 / \sqrt{2}+5 / 2) x_{a}-\frac{6-\sqrt{2}}{4 \sqrt{2}}$ implies $1 / 2 \leq x_{a}$, hence $x_{a}=1 / 2$. For this value of $x_{a}$ the relevant interval conditions for (d) give $3 / 2 \leq F^{*} / t \leq 3 / 2$, so $F^{*} / t=3 / 2$. It is easily check that for this values of $x_{a}$ and $F^{*} / t$ the relevant interval conditions for (c') are fulfilled.

Finally we get

$$
\begin{aligned}
& p=t / 2 \\
& p^{*}=0 \\
& F^{*} / t=3 / 2 \\
& x_{a}=1 / 2
\end{aligned}
$$

which is a particular case of (5) above.

\subsubsection{Cases (d) and (d')}

$$
\begin{aligned}
& p=\frac{p^{*}+F^{*}+t x_{a}}{2} \\
& p^{*}=p-F^{*}
\end{aligned}
$$


This system gives the following solution

$$
\begin{aligned}
& p=t x_{a} \\
& p^{*}=t x_{a}-F^{*}
\end{aligned}
$$

The relevant interval conditions for (d) write $-F^{*}+3 t x_{a} \leq t x_{a}-F^{*} \leq$ $-F^{*}+t\left(2-x_{a}\right)$. It is equivalent to $2 x_{a} \leq 0$, hence $x_{a}=0$. For this value of $x_{a}$ we have $p=0$ and $p^{*}=-F^{*}$. The relevant interval conditions for (d') write $0 \geq F^{*}+t$ or $-1 \geq F^{*} / t$. which is not possible since $F^{*}>0$.

\subsection{Case (e)}

In this case the market share of the on-line firm is zero.

\subsubsection{Cases (e) and (a')}

$$
\begin{aligned}
& p=p^{*}+F^{*}-t\left(1-x_{a}\right) \\
& p^{*} \geq p-F^{*}+t\left(1-x_{a}\right)
\end{aligned}
$$

This gives $p=p^{*}+F^{*}-t\left(1-x_{a}\right)$.

The relevant interval condition for ( $\left.\mathrm{a}^{\prime}\right)$ is $p^{*} \leq F^{*}-t\left(1-x_{a}\right)$. Hence we have $p^{*}+F^{*}-t\left(1-x_{a}\right) \leq F^{*}-t\left(1-x_{a}\right)$ and $p^{*}=0$. Now this induces $p=F^{*}-t\left(1-x_{a}\right)$. The relevant interval condition for (e) writes $F^{*} / t \geq 2-x_{a}$. Thus the positivity requirement for $p$ is trivially satisfied. We then derived the following intersection

$$
\begin{aligned}
& p=F^{*}-t\left(1-x_{a}\right) \\
& p^{*}=0 \\
& F^{*} / t \geq 2-x_{a} .
\end{aligned}
$$

Under these conditions we have $\pi=F^{*}-t\left(1-x_{a}\right)$ and $\pi^{*}=0$. Notice that these conditions encompasse (3) and (4).

\subsubsection{Cases (e) and (b')}

$$
\begin{aligned}
& p=p^{*}+F^{*}-t\left(1-x_{a}\right) \\
& p^{*}=\frac{p-F^{*}+t\left(1-x_{a}\right)}{2}
\end{aligned}
$$

The solution is

$$
\begin{aligned}
& p=F^{*}-t\left(1-x_{a}\right) \\
& p^{*}=0
\end{aligned}
$$

The relevant interval condition for (e) writes again as $F^{*} / t \geq 2-x_{a}$, which implies $p>0$. 
If $0 \leq x_{a} \leq 3 / 2-\sqrt{2}$ the relevant interval conditions for ( $\left.\mathrm{b}^{\prime}\right)$ write $F^{*}-t\left(1-x_{a}\right) \leq F^{*}-t\left(1-x_{a}\right) \leq F^{*}+t\left(1-\sqrt{x_{a}}\right)^{2}$. Which is always fulfilled. The same property holds for the case $3 / 2-\sqrt{2} \leq x_{a} \leq 1 / 2$. We have exactly the same conditions as in (6).

3.5.3 Cases (e) and (c')

$$
\begin{aligned}
& p=p^{*}+F^{*}-t\left(1-x_{a}\right) \\
& p^{*}=\frac{p-F^{*}+t / 2}{2}
\end{aligned}
$$

The solution of this system is

$$
\begin{aligned}
& p=F^{*}-t\left(3 / 2-2 x_{a}\right) \\
& p^{*}=\frac{t\left(2 x_{a}-1\right)}{2}
\end{aligned}
$$

The relevant interval condition for (e) writes $-F^{*}+t\left(2-x_{a}\right) \leq$ $\frac{t\left(2 x_{a}-1\right)}{2}$. It is equivalent to $5 / 2-2 x_{a} \leq F^{*} / t$. The relevant interval conditions for $\left(\mathrm{c}^{\prime}\right)$ are $F^{*}-t\left((\sqrt{2}+1) x_{a}-\frac{1}{\sqrt{2}}\right) \leq F^{*}-t\left(3 / 2-2 x_{a}\right) \leq F^{*}+\frac{t}{2}$. It is equivalent to $1 / 2 \leq x_{a} \leq 1$, hence $x_{a}=1 / 2$. For this value of $x_{a}$ we have $p^{*}=0$ and $p=F^{*}-t / 2$. It is easily checked that we are back to a particular case (5) above.

\subsubsection{Cases (e) and (d')}

$$
\begin{aligned}
& p=p^{*}+F^{*}-t\left(1-x_{a}\right) \\
& p^{*}=p-F^{*}
\end{aligned}
$$

This system implies $t\left(1-x_{a}\right)=0$ which is impossible if $t>0$ and $x_{a} \leq 1 / 2$.

\subsection{Gathering solutions}

- monopoly of local firm

$$
\begin{gathered}
p=F^{*}-t\left(1-x_{a}\right) \\
p^{*}=0 \\
F^{*} / t \geq 2-x_{a}
\end{gathered}
$$

or

$1 \leq F^{*} / t \leq 3 / 2$ and $x_{a}=1 / 2$ 
- duopoly type I

$$
\begin{gathered}
p=\frac{F^{*}+t\left(1+x_{a}\right)}{3} \\
p^{*}=\frac{t\left(2-x_{a}\right)-F^{*}}{3} \\
\left(-1-x_{a}+3 \sqrt{x_{a}}\right) \mathbf{1}_{0 \leq x_{a}<3 / 2-\sqrt{2}}+\left(\left(\frac{3}{\sqrt{2}}+2\right) x_{a}+\frac{1}{2}-\frac{3}{2 \sqrt{2}}\right) \mathbf{1}_{3 / 2-\sqrt{2} \leq x_{a} \leq 1 / 2} \leq F^{*} / t \leq 2-x_{a}
\end{gathered}
$$

- duopoly type II

$$
\begin{gathered}
p=\frac{F^{*}+t / 2}{3} \\
p^{*}=\frac{t-F^{*}}{3} \\
-\frac{1}{2} \leq F^{*} / t \leq \frac{1}{4}-\frac{3}{2 \sqrt{2}}+\frac{3(\sqrt{2}+1) x_{a}}{2}
\end{gathered}
$$

\section{Computation of mixed strategy equilibria}

As the previous section makes clear, the existence of pure strategy Nash equilibrium is not guarantee for the pricing game. As the profit functions are both continuous, we know that mixed strategy Nash Equilibrium always exists. The purpose of this section is to compute some of the mixed strategies equilibria. In the following, the term "randomized" is used to described cases in which at least one price decision is genuinely random (in the sense that it is not a degenerated random variable). The terminology "mixed" encompasses both "randomized" and "pure" strategy cases.

As Osborne and Pitchik (1987) made clear the computation of these equilibria is involving and some of the results rest on computational approach. We also have to tackle with this difficult issue. To this end we choose to study the conditions for existence of "simple" randomized equilibrium of the pricing game. By simple we mean that we try to restrict as far as possible the support of the distributions. We then first examine the conditions under which the on-line firm uses a random pricing rule.

\subsection{Pure/random equilibria}

As the sections 1 and 2 make clear, the cases for a random pricing rule for the on-line firm and a pure pricing strategy for the local firms are as follows:

- $x_{a} \leq 3 / 2-\sqrt{2}$

$$
\begin{aligned}
& p=F^{*}+t\left(1-\sqrt{x_{a}}\right)^{2} \\
& p^{*} \in\left\{p-F^{*},\left(p-F^{*}+t\left(1-x_{a}\right)\right) / 2\right\}
\end{aligned} \Leftrightarrow \begin{aligned}
& p=F^{*}+t\left(1-\sqrt{x_{a}}\right)^{2} \\
& p^{*} \in\left\{t\left(1-\sqrt{x_{a}}\right)^{2}, t\left(1-\sqrt{x_{a}}\right)\right\}
\end{aligned}
$$


- $3 / 2-\sqrt{2} \leq x_{a} \leq 1 / 2$

$p=F^{*}-t\left((\sqrt{2}+1) x_{a}-1 / \sqrt{2}\right)$
$p^{*} \in\left\{\left(p-F^{*}+t / 2\right) / 2,\left(p-F^{*}+t\left(1-x_{a}\right)\right) / 2\right\}$$\quad \Leftrightarrow \quad \begin{aligned} & p=F^{*}-t\left((\sqrt{2}+1) x_{a}-1 / \sqrt{2}\right) \\ & p^{*} \in\left\{t \frac{\sqrt{2}+1}{4}\left(1-2 x_{a}\right), t \frac{\sqrt{2}+2}{4}\left(1-2 x_{a}\right)\right\}\end{aligned}$

Notice that when $x_{a}=3 / 2-\sqrt{2}$ both cases provide the same solutions. We first study the case where $x_{a} \geq 3 / 2-\sqrt{2}$.

4.2 Case $1 / 2 \geq x_{a} \geq 3 / 2-\sqrt{2}$

In this subsection we denote

$$
\begin{aligned}
& p_{0}=F^{*}-t\left((\sqrt{2}+1) x_{a}-1 / \sqrt{2}\right) \\
& p_{1}^{*}=t \frac{\sqrt{2}+1}{4}\left(1-2 x_{a}\right) \\
& p_{2}^{*}=t \frac{\sqrt{2}+2}{4}\left(1-2 x_{a}\right)
\end{aligned}
$$

If $p^{*}=p_{j}^{*}, j=1,2$ the market share of the local firm is given by

$$
\begin{array}{lll}
0 & \text { if } & p \geq p_{j}^{*}+F^{*} \\
2 \frac{F^{*}+p_{j}^{*}-p}{t} & \text { if } & p_{j}^{*}+F^{*} \geq p \geq p_{j}^{*}+F^{*}-t x_{a} \\
x_{a}+\frac{F^{*}+p_{j}^{*}-p}{t} & \text { if } & p_{j}^{*}+F^{*}-t x_{a} \geq p \geq p_{j}^{*}+F^{*}-t\left(1-x_{a}\right) \\
1 & \text { if } & p_{j}^{*}+F^{*}-t\left(1-x_{a}\right) \geq p
\end{array}
$$

If $1 / 2 \geq x_{a} \geq 3 / 2-\sqrt{2}$, we easily derive the following inequalities

$$
\begin{aligned}
& p_{2}^{*}+F^{*} \geq \max \left\{p_{1}^{*}+F^{*}, p_{2}^{*}+F^{*}-t x_{a}\right\} \\
& \max \left\{p_{1}^{*}+F^{*}, p_{2}^{*}+F^{*}-t x_{a}\right\} \geq \min \left\{p_{1}^{*}+F^{*}, p_{2}^{*}+F^{*}-t x_{a}\right\} \\
& \min \left\{p_{1}^{*}+F^{*}, p_{2}^{*}+F^{*}-t x_{a}\right\} \geq p_{1}^{*}+F^{*}-t x_{a} \\
& p_{1}^{*}+F^{*}-t x_{a} \geq p_{2}^{*}+F^{*}-t\left(1-x_{a}\right) \\
& p_{2}^{*}+F^{*}-t\left(1-x_{a}\right) \geq p_{1}^{*}+F^{*}-t\left(1-x_{a}\right)
\end{aligned}
$$

Moreover, $p_{2}^{*}+F^{*}-t x_{a} \leq p_{1}^{*}+F^{*}$ requires $x_{a} \geq 1 / 6$. It is also easy to derive that we have $\min \left\{p_{1}^{*}+F^{*}, p_{2}^{*}+F^{*}-t x_{a}\right\} \geq p_{0} \geq p_{1}^{*}+F^{*}-t x_{a}$.

\subsubsection{Necessary conditions}

Whenever $\min \left\{p_{1}^{*}+F^{*}, p_{2}^{*}+F^{*}-t x_{a}\right\} \geq p \geq p_{1}^{*}+F^{*}-t x_{a}$ the maximum of the profit function must be reached when $p=p_{0}$ for a randomized equilibrium to exist.

In this case, the expected profit is 


$$
\left(1+q^{*}\right) \frac{p}{t}\left(F^{*}+t \frac{\sqrt{2}}{4}-t x_{a} \frac{\sqrt{2}}{2}-\frac{q^{*} t x_{a}}{1+q}+\frac{t}{2(1+q)}-p\right)
$$

where $q^{*}$ is the probability of the event "the on-line firm's price is $p_{1}^{*}$.

As a function of $p$, the expected profit is maximized for

$$
p=\frac{F^{*}}{2}+t \frac{\sqrt{2}}{8}-t x_{a} \frac{\sqrt{2}}{4}-\frac{q^{*} t x_{a}}{2\left(1+q^{*}\right)}+\frac{t}{4\left(1+q^{*}\right)}
$$

There is a unique value of $q^{*}$ such that $p_{0}=F^{*} / 2+t \frac{\sqrt{2}}{8}-t x_{a} \frac{\sqrt{2}}{4}-$ $\frac{q^{*} t x_{a}}{2\left(1+q^{*}\right)}+\frac{t}{4\left(1+q^{*}\right)}$. This value of $q^{*}$ satisfies:

$$
q^{*}\left(\frac{F^{*}}{t}-x_{a}(3 / \sqrt{2}+1)+\frac{3}{2 \sqrt{2}}\right)=-\frac{F^{*}}{t}+x_{a}(3 / \sqrt{2}+2)-\frac{3}{2 \sqrt{2}}+\frac{1}{2}
$$

The condition $0 \leq q^{*} \leq 1$ is equivalent to

$$
x_{a}(3 / \sqrt{2}+2)-\frac{3}{2 \sqrt{2}}+\frac{1}{2} \geq \frac{F^{*}}{t} \geq x_{a}(3 / \sqrt{2}+3 / 2)-\frac{3}{2 \sqrt{2}}+\frac{1}{4}
$$

It coincides exactly with the cases where the pure strategy equilibrium does not exist when $1 / 2 \geq x_{a} \geq 3 / 2-\sqrt{2}$. We then derived the following necessary conditions for our equilibria

$$
\begin{aligned}
& p=F^{*}-t\left((\sqrt{2}+1) x_{a}-1 / \sqrt{2}\right) \\
& p^{*} \in\left\{t \frac{\sqrt{2}+1}{4}\left(1-2 x_{a}\right), t \frac{\sqrt{2}+2}{4}\left(1-2 x_{a}\right)\right\} \\
& \mathbf{P}\left(\tilde{p}^{*}=t \frac{\sqrt{2}+1}{4}\left(1-2 x_{a}\right)\right)=\frac{t x_{a}(6+4 \sqrt{2})+t(\sqrt{2}-3)-2 \sqrt{2} F^{*}}{2 \sqrt{2} F^{*}-t x_{a}(6+2 \sqrt{2})+3 t} \\
& x_{a}(3 / \sqrt{2}+2)-\frac{3}{2 \sqrt{2}}+\frac{1}{2} \geq \frac{F^{*}}{t} \geq x_{a}(3 / \sqrt{2}+3 / 2)-\frac{3}{2 \sqrt{2}}+\frac{1}{4}
\end{aligned}
$$

where $\tilde{p}^{*}$ stands for the random price of the on-line firm.

The above conditions are not sufficient. Indeed, section 1 guarantees that if the on-line firm does not randomize then the profit function of the local firm is strictly concave. However, when the on-line firm uses a random pricing rule, the expected profit function of the local firm is no longer strictly concave. We then have to study the conditions under which the rule $p=p_{0}$ provides a genuine global maximum.

A genuine analytical study of the sufficiency conditions is very long. However, a numerical approach is not difficult. Indeed, the expected profit of the local firm is piecewise quadratic, thus all the candidates for local maxima may be explicitly computed as functions of $x_{a}$ and $F^{*} / t$. We proceed numerically to compare these local maxima. It then appears that the 
above local maximum may coincide with the solution $p=\frac{p_{2}^{*}+F^{*}+x_{a}}{2}$ or $p=$ $p_{2}^{*}+F^{*}-x_{a}$.

\subsection{Case $x_{a} \leq 3 / 2-\sqrt{2}$}

We first make explicit the game played by the local firm if the on-line firm random drawing selects $p^{*}=t\left(1-\sqrt{x_{a}}\right)^{2}$. As before, we have

$$
\begin{array}{lll}
\Delta\left(p, x_{a}\right)=0 & \text { if } & p \geq F^{*}+t\left(1-\sqrt{x_{a}}\right)^{2} \\
\Delta\left(p, x_{a}\right)=2 \frac{F^{*}+t\left(1-\sqrt{x_{a}}\right)^{2}-p}{t} & \text { if } & F^{*}+t\left(1-\sqrt{x_{a}}\right)^{2} \geq p \geq F^{*}+t\left(1-2 \sqrt{x_{a}}\right) \\
\Delta\left(p, x_{a}\right)=x_{a}+\frac{F^{*}+t\left(1-\sqrt{x_{a}}\right)^{2}-p}{t} & \text { if } & F^{*}+t\left(1-2 \sqrt{x_{a}}\right) \geq p \geq F^{*}-2 t\left(\sqrt{x_{a}}-x_{a}\right) \\
\Delta\left(p, x_{a}\right)=1 & \text { if } & F^{*}-2 t\left(\sqrt{x_{a}}-x_{a}\right) \geq p
\end{array}
$$

If the random draw selects $p^{*}=t\left(1-\sqrt{x_{a}}\right)$, we compute

$$
\begin{array}{lll}
\Delta\left(p, x_{a}\right)=0 & \text { if } & p \geq F^{*}+t\left(1-\sqrt{x_{a}}\right) \\
\Delta\left(p, x_{a}\right)=2 \frac{F^{*}+t\left(1-\sqrt{x_{a}}\right)-p}{t} & \text { if } & F^{*}+t\left(1-\sqrt{x_{a}}\right) \geq p \geq F^{*}+t\left(1-\sqrt{x_{a}}-x_{a}\right) \\
\Delta\left(p, x_{a}\right)=x_{a}+\frac{F^{*}+t\left(1-\sqrt{x_{a}}\right)-p}{t} & \text { if } & F^{*}+t\left(1-\sqrt{x_{a}}-x_{a}\right) \geq p \geq F^{*}-t\left(\sqrt{x_{a}}-x_{a}\right) \\
\Delta\left(p, x_{a}\right)=1 & \text { if } & F^{*}-t\left(\sqrt{x_{a}}-x_{a}\right) \geq p
\end{array}
$$

First remark that $0 \leq x_{a} \leq 3 / 2-\sqrt{2}$ implies

$$
\begin{gathered}
F^{*}+t\left(1-\sqrt{x_{a}}\right) \geq F^{*}+t\left(1-\sqrt{x_{a}}-x_{a}\right) \geq F^{*}+t\left(1-\sqrt{x_{a}}\right)^{2} \geq F^{*}+t\left(1-2 \sqrt{x_{a}}\right)>F^{*}- \\
t\left(\sqrt{x_{a}}-x_{a}\right) \geq F^{*}-2 t\left(\sqrt{x_{a}}-x_{a}\right)
\end{gathered}
$$

If we denote $q=\mathbf{P}\left(p^{*}=t\left(1-\sqrt{x_{a}}\right)^{2}\right)$ the expected market share of the local firm $\Delta\left(p, x_{a}, q\right)$ is given by

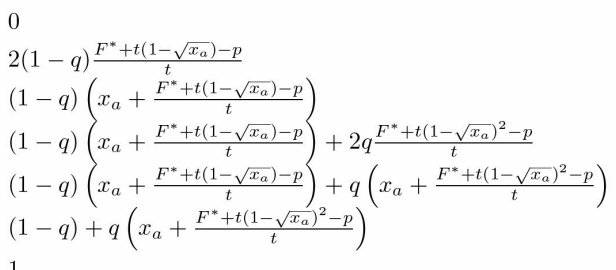

The expected profit of the local firm is $\Delta\left(p, x_{a}, q\right) p$. We now detail the conditions under which $p=F^{*}+t\left(1-\sqrt{x_{a}}\right)^{2}$ is the optimal choice. The expected profit for this choice of price is is $(1-q) \sqrt{x_{a}}\left(F^{*}+t\left(1-\sqrt{x_{a}}\right)^{2}\right)$. 
For this quantity to be positive, we need

$$
F^{*} / t \geq-\left(1-\sqrt{x_{a}}\right)^{2}
$$

Consider the situation where $p$ belongs to an arbitrarily small neighborhood of $F^{*}+t\left(1-\sqrt{x_{a}}\right)^{2}$.

When $p \leq F^{*}+t\left(1-\sqrt{x_{a}}\right)^{2}$, the expected profit function is

$$
(1-q) p\left(x_{a}+\frac{F^{*}+t\left(1-\sqrt{x_{a}}\right)-p}{t}\right)+2 q p \frac{F^{*}+t\left(1-\sqrt{x_{a}}\right)^{2}-p}{t}
$$

and when $p \geq F^{*}+t\left(1-\sqrt{x_{a}}\right)^{2}$ the expected profit function is

$$
(1-q) p\left(x_{a}+\frac{F^{*}+t\left(1-\sqrt{x_{a}}\right)-p}{t}\right)
$$

Thus, a necessary condition for $p=F^{*}+t\left(1-\sqrt{x_{a}}\right)^{2}$ to be a maximum of the expected profit is

$$
\begin{aligned}
& \frac{\partial}{\partial p}\left\{(1-q) p\left(x_{a}+\frac{F^{*}+t\left(1-\sqrt{x_{a}}\right)-p}{t}\right)+2 q p \frac{F^{*}+t\left(1-\sqrt{x_{a}}\right)^{2}-p}{t}\right\}_{p=F^{*}+t\left(1-\sqrt{x_{a}}\right)^{2}} \geq 0 \\
& \frac{\partial}{\partial p}\left\{(1-q) p\left(x_{a}+\frac{F^{*}+t\left(1-\sqrt{x_{a}}\right)-p}{t}\right)\right\}_{p=F^{*}+t\left(1-\sqrt{x_{a}}\right)^{2}} \leq 0
\end{aligned}
$$

If we consider only the case for mixed equilibrium, we have $0<q<1$ and these inequalities are then equivalent to

$$
\begin{gathered}
(1-q)\left(x_{a}+\frac{F^{*}+t\left(1-\sqrt{x_{a}}\right)-\left(F^{*}+t\left(1-\sqrt{x_{a}}\right)^{2}\right)}{t}\right)+2 q \frac{F^{*}+t\left(1-\sqrt{x_{a}}\right)^{2}-\left(F^{*}+t\left(1-\sqrt{x_{a}}\right)^{2}\right)}{t}-\frac{(1-q)\left(F^{*}+t\left(1-\sqrt{x_{a}}\right)^{2}\right)}{t}-\frac{2 q\left(F^{*}+t(1-\sqrt{x}\right.}{t} \\
x_{a}+\frac{F^{*}+t\left(1-\sqrt{x_{a}}\right)-\left(F^{*}+t\left(1-\sqrt{x_{a}}\right)^{2}\right)}{t}-\frac{F^{*}+t\left(1-\sqrt{x_{a}}\right)^{2}}{t} \leq 0 \\
(1-q)\left(x_{a}+1-\sqrt{x_{a}}-\left(1-\sqrt{x_{a}}\right)^{2}\right)-(1+q)\left(F^{*} / t+\left(1-\sqrt{x_{a}}\right)^{2}\right) \geq 0 \\
x_{a}+1-\sqrt{x_{a}}-\left(1-\sqrt{x_{a}}\right)^{2}-F^{*} / t-\left(1-\sqrt{x_{a}}\right)^{2} \leq 0 \\
(1-q)\left(x_{a}+1-\sqrt{x_{a}}-1+2 \sqrt{x_{a}}-x_{a}\right)-(1+q)\left(F^{*} / t+\left(1-\sqrt{x_{a}}\right)^{2}\right) \geq 0 \\
x_{a}+1-\sqrt{x_{a}}-1+2 \sqrt{x_{a}}-x_{a}-F^{*} / t-\left(1-\sqrt{x_{a}}\right)^{2} \leq 0 \\
(1-q) \sqrt{x_{a}}-(1+q)\left(F^{*} / t+1-2 \sqrt{x_{a}}+x_{a}\right) \geq 0 \\
-1+3 \sqrt{x_{a}}-x_{a}-F^{*} / t \leq 0 \\
-F^{*} / t-1+3 \sqrt{x_{a}}-x_{a}-q\left(F^{*} / t+1-\sqrt{x_{a}}+x_{a}\right) \geq 0 \\
-1+3 \sqrt{x_{a}}-x_{a} \leq F^{*} / t \\
-F^{*} / t-1+3 \sqrt{x_{a}}-x_{a} \geq q\left(F^{*} / t+1-\sqrt{x_{a}}+x_{a}\right) \\
-1+3 \sqrt{x_{a}}-x_{a} \leq F^{*} / t \\
\frac{-F^{*} / t-1+3 \sqrt{x_{a}}-x_{a}}{F^{*} / t+1-\sqrt{x_{a}}+x_{a}} \leq q \\
-1+3 \sqrt{x_{a}}-x_{a} \leq F^{*} / t \\
F^{*} / t+1-\sqrt{x_{a}}+x_{a} \leq 0 \\
\frac{-F^{*} / t-1+3 \sqrt{x_{a}}-x_{a}}{F^{*} / t+1-\sqrt{x_{a}}+x_{a}} \geq q \\
-1+3 \sqrt{x_{a}}-x_{a} \geq F^{*} / t \\
F^{*} / t \geq-1+\sqrt{x_{a}}-x_{a} \\
\end{gathered}
$$




\subsection{Gathering solutions}

The randomized equilibria are then given by the following conditions

- $x_{a} \leq 3 / 2-\sqrt{2}$

$$
\begin{aligned}
& p=F^{*}+t\left(1-\sqrt{x_{a}}\right)^{2} \\
& p^{*} \in\left\{t\left(1-\sqrt{x_{a}}\right)^{2}, t\left(1-\sqrt{x_{a}}\right)\right\} \\
& \frac{-1-x_{a}+3 \sqrt{x_{a}}-F^{*} / t}{F^{*} / t+x_{a}+1-\sqrt{x_{a}}} \leq \mathbf{P}\left(p^{*}=t\left(1-\sqrt{x_{a}}\right)^{2}\right) \leq \frac{3 \sqrt{x_{a}}-F^{*} / t-1}{F^{*} / t+1-\sqrt{x_{a}}} \\
& -1-x_{a}+3 \sqrt{x_{a}} \geq F^{*} / t \geq-\left(1-\sqrt{x_{a}}\right)^{2}
\end{aligned}
$$

- $3 / 2-\sqrt{2} \leq x_{a} \leq 1 / 2$

$$
\begin{aligned}
& p=F^{*}-t\left((\sqrt{2}+1) x_{a}-1 / \sqrt{2}\right) \\
& p^{*} \in\left\{t \frac{\sqrt{2}+1}{4}\left(1-2 x_{a}\right), t \frac{\sqrt{2}+2}{4}\left(1-2 x_{a}\right)\right\} \\
& \mathbf{P}\left(p^{*}=t \frac{\sqrt{2}+1}{4}\left(1-2 x_{a}\right)\right)=\frac{t x_{a}(6+4 \sqrt{2})+t(\sqrt{2}-3)-2 \sqrt{2} F^{*}}{2 \sqrt{2} F^{*}-t x_{a}(6+2 \sqrt{2})+3 t} \\
& x_{a}(3 / \sqrt{2}+2)-\frac{3}{2 \sqrt{2}}+\frac{1}{2} \geq \frac{F^{*}}{t} \geq x_{a}(3 / \sqrt{2}+3 / 2)-\frac{3}{2 \sqrt{2}}+\frac{1}{4}
\end{aligned}
$$

